\title{
Sharp interface limits of phase-field models
}

\author{
K. R. Elder ${ }^{1, *}$ Martin Grant, ${ }^{2}$ Nikolas Provatas,${ }^{2,3}$ and J. M. Kosterlitz ${ }^{4}$ \\ ${ }^{1}$ Department of Physics, Oakland University, Rochester, Michigan 48309-4487 \\ ${ }^{2}$ Physics Department, Rutherford Building, 3600 rue University, McGill University, Montréal, Québec, Canada H3A $2 T 8$ \\ ${ }^{3}$ Pulp and Paper Research Institute of Canada, 570 St. Jean Blvd, Montréal, Québec, Canada H9R-3J9 \\ ${ }^{4}$ Department of Physics, Brown University, Providence, Rhode Island 02912
}

(Received 25 October 2000; published 19 July 2001)

\begin{abstract}
The use of continuum phase-field models to describe the motion of well-defined interfaces is discussed for a class of phenomena that includes order-disorder transitions, spinodal decomposition and Ostwald ripening, dendritic growth, and the solidification of eutectic alloys. The projection operator method is used to extract the "sharp-interface limit" from phase-field models which have interfaces that are diffuse on a length scale $\xi$. In particular, phase-field equations are mapped onto sharp-interface equations in the limits $\xi \kappa \ll 1$ and $\xi v / D$ $\ll 1$, where $\kappa$ and $v$ are, respectively, the interface curvature and velocity and $D$ is the diffusion constant in the bulk. The calculations provide one general set of sharp-interface equations that incorporate the GibbsThomson condition, the Allen-Cahn equation, and the Kardar-Parisi-Zhang equation.
\end{abstract}

DOI: 10.1103/PhysRevE.64.021604 PACS number(s): 81.30.Hd, 05.70.Ln, 64.60.My, 64.60.Cn

\section{INTRODUCTION}

Many inhomogeneous systems involve domains of welldefined phases separated by thin interfaces. These include nonequilibrium systems undergoing phase separation by spinodal decomposition or by nucleation and growth [1], or where solidification occurs by dendritic growth [2], or by the growth of eutectic crystals [3]. The phenomenological description of these phenomena involves the motion of welldefined sharp interfaces. The origin of such descriptions is often transparent, being obtained by symmetry arguments and common sense. Nevertheless the properties of sharpinterface models can be quite subtle as is the case for dendritic growth.

Unfortunately sharp-interface models are difficult to simulate since this usually involves solving a diffusion equation subject to moving boundary conditions at the interfaces. A more convenient approach is to simulate models which describe the bulk phases as well as the interface structure. While these models are wasteful in terms of simulating bulk regions [4], no explicit boundary tracking is needed. This is the key element to a popular method for studying systems out of equilibrium, called "phase-field" modeling. In such an approach one or more continuous fields which are functions of space $\vec{r}$ and time $t$ are introduced to describe the phases present. Typically these fields vary slowly in bulk regions and rapidly on length scales of the order of the correlation length, $\xi$, near interfaces. The free energy functional $\mathcal{F}$ determines the phase behavior and, with the equations of motion, gives a complete description. In other contexts, such as critical dynamics $[1,5]$, the fields are the order parameters distinguishing the phases. In a binary alloy, for example, the local concentration or sublattice concentration can be described by such fields. The ideas involved in this approach have a long history, going back to van der Waals [6]. Within

\footnotetext{
*Electronic address: elder@ oakland.edu
}

the materials community, the use of continuum field models is associated particularly with the work of Cahn and collaborators $[7,8]$.

Phase-field models provide a description at length scales greater than some microscopic scale of the order of a lattice spacing, but are not necessarily appropriate for a particular system. These models apply to a large number of microscopic systems only when $\xi$ is much larger than any particular microscopic length, such as the lattice spacing, involved in the surface structure. Interpreted in the sense of describing the universal features of many microscopic models, $\xi$ is a mesoscopic length representative of the microscopic structure. In a similar manner, sharp-interface models apply to a large number of continuum field models of pattern formation or phase separation in the limit that the length scales defined by the patterns are much larger than $\xi$. An important difference exists, however, in the construction of the two approaches. Standard sharp-interface models are constructed from phenomenological descriptions of interfaces, while phase-field models can be constructed to obey explicitly the fundamental principles of statistical mechanics. In principle, a phase-field model will describe a system in thermal and mechanical equilibrium with its environment and its evolution from some nonequilibrium state towards its ultimate equilibrium state and must contain a sharp- or thin-interface description as a particular limit.

While continuum phase-field models provide a fundamental approach which is clear and workable, it is important to establish the connection between this description and the sharp-interface description. The main difficulty which arises is how to take account of the finite thickness $\xi$ of the diffuse interface of the continuum model. There has been a great deal of discussion in the literature on how this process is to be undertaken. Some workers have extracted the interface equations by taking the limit where the interface width of the phase field model goes to zero [9] for the Stefan problem of a pure material. This approach is not very useful since the interface width is always finite. More recently these calculations have been extended for special choices of the free en- 
ergy functional $\mathcal{F}$ to include an interface of nonzero width $[10,11]$.

The purpose of this paper is to provide a clear and detailed derivation of the sharp interface equations. The approach follows the projection operator method of Kawasaki and Ohta [12], is generally applicable, and eliminates the counterintuitive necessity of limiting the derivation of the sharp interface limit to some artificial form of the free energy functional $\mathcal{F}$ [10]. This general calculation provides one set of equations that relate the parameters of the phase-field equations to those of the sharp interface equations for a broad class of phenomena including order-disorder transitions, dendritic growth, phase separation in binary alloys, eutectic growth, and surface roughening. In particular, thermodynamic consistency is automatic in the present approach for nonzero interface widths. This is in contrast to the unphysical approach of taking the limit of a zero interface width, which requires fine tuning of the free energy to obtain a thermodynamically consistent theory.

The structure of the paper is as follows. In Sec. II a general discussion of out of equilibrium interface phenomena from both the phase-field and from the sharp-interface approach is given. The advantages of the former from a statistical mechanics viewpoint are also pointed out. This contains three subsections: Sec. II A in which systems with a nonconserved field only are described, Sec. II B where the inclusion of a conserved field representing temperature or chemical concentration is discussed, and Sec. IIC in which the free energy or appropriate thermodynamic potential and dynamical equations for a eutectic system with both conserved and nonconserved fields are introduced. Possible equilibrium phase diagrams are also discussed. Next, in Sec. III, sharpinterface equations are obtained by projecting the dynamics of the conserved and nonconserved fields onto the interface by a projection operator method [12]. This rather technical derivation involves an "inner" expansion near the interface in Sec. III A in terms of curvilinear coordinates and an "outer" expansion far from the interface, Sec. III B. In Sec. III C the inner and outer solutions are matched in a region where both are valid. An ambiguity in the definition of the interface position is exploited by defining this position as the Gibbs surface which eliminates the ambiguity. This is a solvability condition. In Sec. IV expressions are given for quantities such as the capillary length, $d_{0}$, the surface tension $\sigma$, and the kinetic undercooling coefficient $\beta$ which are parameters of the sharp-interface equations in terms of the free energy functional $\mathcal{F}$. It is also shown that $\beta>0$ for nonconserved model A dynamics, $\beta<0$ for conserved model B dynamics, and is of either sign for the physically important case of model C dynamics [5]. In Sec. V, the dynamics of fluctuations about almost planar interfaces is discussed in terms of the sharp-interface equations of Sec. III in three cases. (i) In Sec. V A, the noiseless Kardar-Parisi-Zhang equation is derived for a nonconserved field. (ii) In Sec. V B the dispersion relation $\omega(k)$ is derived for spinodal decomposition in pure conserved model B dynamics and the crossover from $\omega \propto-k^{3}$ at small $k$ to $\omega \propto-k^{2}$ at large $k$. The influence of the kinetic undercooling coefficient $\beta<0$ is also discussed. (iii) Then, in Sec. V C, the physically important case of coupled conserved and nonconserved fields describing the invasion of a supersaturated liquid phase by a stable phase is discussed. Also, the widely used phenomenological model of solidification is discussed as a special case of the more general model considered here and several results which were previously derived numerically are rederived analytically. A brief summary of the paper is in Sec. VI. Several technical details of the sometimes rather tedious mathematical formalism are relegated to the Appendixes.

\section{DESCRIBING INTERFACE PHENOMENA USING PHASE-FIELD MODELS}

\section{A. Nonconserved field}

To see the relationship between the two approaches, it is useful to construct the simplest equilibrium and nonequilibrium descriptions of surfaces by both methods. The main ideas developed below appear in the more general case discussed in the following sections. These ideas are present in the work by Allen and Cahn [8] on the motion of antiphase boundaries in the kinetics of an order-disorder transition in a binary alloy. This work recovers the sharp-interface description from the phase-field model in the appropriate limit and predicts an experimentally testable consequence of a finite $\xi$.

First, consider a surface in equilibrium. The reason why droplets are spheres and interfaces are locally flat is that these shapes minimize surface area. The excess free energy of a surface is proportional to its area $A$

$$
\Delta \mathcal{F}=\sigma A,
$$

where the proportionality constant $\sigma$ is the surface tension. This simple common sense approach is the essence of the sharp-interface model.

Contrast this with the phase-field approach. The free energy functional $\mathcal{F}(\psi)$ for the scalar order parameter $\psi(\vec{r})$ has the following familiar form, consistent with reflection symmetry $\mathcal{F}(\psi)=\mathcal{F}(-\psi)$

$$
\mathcal{F}\{\psi\}=\int d \vec{r}\left[\frac{1}{2} K_{\psi}(\vec{\nabla} \psi)^{2}+f(\psi)\right],
$$

where $K_{\psi}>0$ so that the square gradient interaction gives the free energy cost for inhomogeneities and the local bulk free energy has a double well form satisfying $f(\psi)=f(-\psi)$. An example is the $\psi^{4}$ form

$$
f=-\frac{a}{2} \psi^{2}+\frac{b}{4} \psi^{4}
$$

where $a \propto\left(T_{o}-T\right)>0$ with $T_{o}$ the mean field critical temperature and $b>0$ is a constant.

The motivation for employing such free energy functionals is as follows. First, the free energy functional is constructed of local or bulk terms, $f(\psi)$, which interact through the gradient term. Second $\mathcal{F}$ must be an analytic function since $\mathcal{F} \psi(\vec{r})]$ is the free energy of a particular configuration of $\psi(\vec{r})$ which has spatial variations on scales larger than some microscopic cutoff length which is of the order of an 
interparticle separation or a lattice spacing $a_{0} . \mathcal{F}$ describes a mesoscopic system and should not be confused with the true thermodynamic free energy, $F$ $\left.\equiv-k_{B} T \ln \Sigma_{\psi} \exp (-\mathcal{F} \psi] / k_{B} T\right)$, where $k_{B}$ is Boltzmann's constant, which is nonanalytic at a phase transition [13]. The square gradient term is the simplest way for the model to be well defined on small length scales. Higher-order gradient terms, such as $K_{4}\left(\nabla^{2} \psi\right)^{2}$ could, in principle, describe correlations on still smaller scales, such as the internal structure of the interface. This is analogous to what is described by the Percus-Yevick or hypernetted-chain theories of dense fluids [14]. In the absence of any specific experimental motivation, however, terms proportional to $K_{4}$ may be ignored. From the point of view of identifying and studying a well-defined microscopic model, the square gradient free energy provides a complete description. Alternatively, the square gradient theory describes a large class of microscopic models provided we consider mesoscopic scales $\xi(K) / a_{o} \gg 1$.

It is particularly convenient that the microscopic phasefield description is so "close" to mean field theory. In that approximation, $f$ is simply the bulk free energy density. In practice, the form of the free energy functional $\mathcal{F}$ can be constructed straightforwardly with reference to the phase diagram of interest. While one can also construct microscopic lattice gas models of phenomena such as phase separation and dendritic solidification [15], using similar arguments of universality and simplicity, such models do not have this convenient feature.

If fluctuations are small, the equilibrium behavior of the model is determined by the mean field approximation

$$
\frac{\delta \mathcal{F}}{\delta \psi}=-K_{\psi} \nabla^{2} \psi+\frac{\partial f}{\partial \psi}=0 .
$$

The homogeneous bulk solutions, valid well below $T_{c}$, are given by $\partial f / \partial \psi_{e q}=0$ and are equal to

$$
\psi= \pm \psi_{e q}= \pm \sqrt{a / b}
$$

for the bulk free energy given in Eq. (3). Fluctuations around the bulk solutions satisfy

$$
\left\langle\left[\psi(\vec{r})-\psi_{e q}\right]\left[\psi(0)-\psi_{e q}\right]\right\rangle \sim e^{-r / \xi}
$$

for $r \gg \xi$, where $\xi=\sqrt{K_{\psi} / 2 a}$ and $\langle\cdots\rangle$ represents an ensemble average.

Now consider a system with a flat interface located at $y$ $=0$ when $\psi(\vec{r})$ depends only on $y$ and the interface profile $\psi^{\text {in }}(y)$ is the solution of

$$
-K_{\psi} \frac{\partial^{2} \psi^{\text {in }}}{\partial y^{2}}+\frac{\partial f}{\partial \psi^{\text {in }}}=0
$$

with $\psi^{\text {in }}( \pm \infty)= \pm \psi_{e q}$. Solving by quadratures gives

$$
\frac{1}{2} K_{\psi}\left(\frac{\partial \psi^{\text {in }}}{\partial y}\right)^{2}=f\left(\psi^{\text {in }}\right)
$$

from which Eq. (1) follows immediately. In contrast to the sharp-interface approach, this yields an explicit form for the surface tension

$$
\sigma_{\psi}=K_{\psi} \int d y\left(\frac{\partial \psi^{\text {in }}}{\partial y}\right)^{2}
$$

For the particular form of $\mathcal{F}(\psi)$ of Eqs. (2) and (3), one finds

$$
\psi^{\text {in }}(y)=\psi_{e q} \tanh \left(\frac{y}{2 \xi}\right)
$$

and

$$
\sigma_{\psi}=\frac{2 K_{\psi} \psi_{e q}^{2}}{3 \xi}
$$

The contrast between the sharp-interface model and the phase-field model is revealing. Both give the same macroscopic description but the phase field approach gives a complete description which is accurate at large length scales and interpolates down to the smallest length scales without destroying any important physics [13].

Now consider a sharp interface that is in local but not global equilibrium due to a gentle curvature. For simplicity consider phenomena where the order parameter is not conserved, such as occurs when a binary alloy undergoes an order-disorder transition and $\psi$ is the sublattice concentration. The interface moves to locally reduce the surface area and surface free energy with an interface velocity $v$. Expanding $v$ in a Taylor series in powers of $\kappa$ gives

$$
v=-\nu \kappa
$$

to lowest order in the curvature. This is the sharp-interface theory for the motion of anti-phase boundaries. Note that, since $\nu$ is the only coefficient which enters the theory and has dimensions of a diffusion constant, any time-dependent length $R(t)$ must satisfy

$$
R(t)=(\nu t)^{n}
$$

by dimensional analysis, where the growth exponent

$$
n=1 / 2
$$

Such an approach was first done by Lifshitz [16] and by Turnbull [17]. The sharp-interface treatment alone cannot predict the value of $\nu$. An additional argument, which turns out to be incorrect for the motion of antiphase boundaries, was used to predict $\nu \propto \sigma_{\psi} \Gamma_{\psi}$, where $\Gamma_{\psi}$ is a mobility.

A first principles approach to this phenomenon is due to Allen and Cahn [8]. Neglecting noise, the equation of motion for the nonconserved sublattice concentration is $[1,5,8]$

$$
\frac{\partial \psi}{\partial t}=-\Gamma_{\psi} \frac{\delta \mathcal{F}}{\delta \psi}=-\Gamma_{\psi}\left[-K_{\psi} \nabla^{2} \psi+\frac{\partial f}{\partial \psi}\right] .
$$


Allen and Cahn denoted the position of the antiphase boundary by a curved, time-dependent interface $u(\vec{r}, t)=0$. They then looked for solutions of the form $\psi(\vec{r}, t)=\psi^{\mathrm{in}}[u(\vec{r}, t)]$. This gives

$$
\frac{\partial \psi}{\partial t}=-v \frac{\partial \psi^{\text {in }}}{\partial u}=-\Gamma_{\psi}\left[-K_{\psi} \frac{\partial^{2} \psi^{\text {in }}}{\partial u^{2}}-K_{\psi} \kappa \frac{\partial \psi^{\text {in }}}{\partial u}+\frac{\partial f}{\partial \psi}\right],
$$

where $\kappa=-\vec{\nabla} \cdot \hat{n}$ with $\hat{n}=\vec{\nabla} u /|\vec{\nabla} u|$ the unit vector normal to the interface and $u(\vec{r}, t)$ a coordinate in the direction $\hat{n}$. Eliminating $\partial f / \partial \psi^{\text {in }}$ using Eq. (7) gives Eq. (12) where, in contrast to the sharp interface theory, one obtains an explicit expression for the transport coefficient,

$$
\nu=K_{\psi} \Gamma_{\psi} .
$$

Results in the presence of stochastic noise have been obtained by many authors, particularly Bausch, Domb, Janssen, and Zia [18] and by Kawasaki and Ohta [12].

Although both approaches correctly find that the normal velocity is proportional to the curvature, the Allen-Cahn result for $\nu$ [8] is noteworthy. The earlier theory, which argued $\nu \propto \sigma_{\psi} \Gamma_{\psi}$ implies a strong dependence of the velocity $v$ on interfacial thickness since $\sigma \propto 1 / \xi$, from Eq. (11). In contrast, Allen and Cahn predict that $\nu$ is independent of interfacial thickness. This was clearly demonstrated in an experiment by Pindak et al. [19] where they studied orientational patterns in freely suspended dipolar smectic $\mathrm{C}$ liquid crystal films. Since their smectic $\mathrm{C}$ films have a permanent electric dipole moment of magnitude $P$, the director angle $\phi$ can be oriented with an electric field of magnitude $E$. The free energy is

$$
\mathcal{F}=\int d \vec{r}\left[\frac{1}{2} K(\nabla \phi)^{2}-E P \cos \phi\right]
$$

so the width of the interface,

$$
\xi \propto 1 / \sqrt{E}
$$

can be varied easily. The experiments directly verify that the size of a domain of stable orientation grows as $R(t)$ $=(\nu t)^{1 / 2}$, where $\nu$ is independent of interface width in accord with the prediction of Allen and Cahn [8]. In addition, the experiments show that the sharp-interface result, $v \propto \kappa$, is independent of interface width provided $\xi / R \ll 1$.

It is straightforward to include an external field $c$ coupling linearly to $\psi$. Then $\mathcal{F} \rightarrow \mathcal{F}+\mathcal{F}_{\text {ext }}$ with

$$
\mathcal{F}_{\text {ext }}=-\int d \vec{r} c \psi
$$

and one of the phases becomes metastable depending on the sign of $c$, so that the interface translates even if it is flat. Hence

$$
v=v_{0}(c)-\nu \kappa
$$

with $v_{0}(c) \propto c$. This allows one to simulate KolmogorovAvrami-Johnson-Mehl growth of droplets [20] and, in the presence of noise, Kardar-Parisi-Zhang dynamic roughening [21].

\section{B. Including a conserved field}

Other processes can be simulated when one of the phases is metastable and the growth of the stable from the metastable phase is controlled by a conservation law. This describes solidification of a metastable supercooled liquid phase and the growth of the stable solid is limited by the diffusion of latent heat from the surface of the moving solid front. The external field $c$ is then proportional to the latent heat. In the sharp-interface formulation, $c$ obeys a diffusion equation in the bulk phases

$$
\frac{\partial c}{\partial t}=D_{c} \nabla^{2} c
$$

where $D_{c}$ is a diffusion constant.

The steady state velocity $v$ of the interface is given by integrating Eq. (22) across the interface to obtain

$$
v \propto \hat{n} \cdot\left(\left.D_{c}^{+} \vec{\nabla} c\right|^{+}-\left.D_{c}^{-} \vec{\nabla} c\right|^{-}\right),
$$

where the superscripts \pm refer to the values of the normal gradient of $c$ on either side of the interface. The condition of local equilibrium at the interface is

$$
\delta c \propto \kappa,
$$

which is a Gibbs-Thompson condition relating the local excess concentration $\delta c$ to the curvature. This says that excess external driving force is balanced by the curvature $\kappa$. In regimes of high undercooling, this is sometimes supplemented by an additive term proportional to $v$, describing kinetic undercooling.

To study this by a phase-field approach, let $c$ be a continuous function of space and time which is conserved:

$$
\frac{\partial c(\vec{r}, t)}{\partial t}=\Gamma_{c} \nabla^{2} \frac{\delta \mathcal{F}}{\delta c}
$$

where $\Gamma_{c}$ is the mobility of the field $c$. For the model to be well defined, a self-energy for $c$ or a positive additive contribution to $\mathcal{F}$ of the form

$$
\mathcal{F}_{c} \propto \int d \vec{r}\left[\frac{1}{2} c^{2}\right]
$$

must be included. Within a mean field approximation, this gives a homogeneous equilibrium solution $c \propto \psi$. Note that the interface invades the metastable phase because of $\mathcal{F}_{\text {ext }}$ but it must also satisfy the conservation of $c$ as defined by Eqs. (25) and (26). This implies that the interface deforms into a parabolic shape, dumping excess $c$ to the sides while propagating forward at a constant velocity. The parabolic shape has a constant growth velocity in the forward direction, satisfying the fact that the system is driven with a con- 
stant thermodynamic force, while lateral growth has a velocity $\sim t^{-1 / 2}$, thereby satisfying the conserved diffusion equation for $c$.

It turns out that, although this is the right approach, the implementation needs some fine tuning. First, when considering dendritic growth, the theory of microscopic solvability [22] has shown that dendrites require an anisotropic surface tension to be well defined. Hence one must let $K$ $\rightarrow K(\vec{\nabla} \psi /|\nabla \psi|)$ in some convenient prescribed way. Next, in a very useful paper, Kobayashi [23] has noted that, to keep the equilibrium solutions $\psi_{e q}$ from shifting appreciably when $c$ is applied, $\mathcal{F}_{\text {ext }}$ of Eq. (20) should be modified to

$$
\mathcal{F}_{\text {ext }}=-\int d \vec{r} c \Psi(\psi),
$$

where $\Psi(\psi)$ is an odd function of $\psi$ satisfying

$$
\partial \Psi / \partial \psi_{e q}=0
$$

For example, if $\psi_{e q}= \pm 1$, one can choose $\partial \Psi / \partial \psi=(1$ $\left.-\psi^{2}\right)^{2}$. Other forms are possible. Finally, one can choose to make the $c$ field phase separate by replacing the self-energy term in the free energy of Eq. (26) with a double well form, analogous to Eq. (3). This permits the study of eutectic crystallization [3].

\section{The free energy functional}

Consider two fields, a nonconserved phase field $\psi$ and a conserved field $c$. The phase field distinguishes between, for example, liquid and solid phases and the $c$ field can be taken as a concentration. The free energy functional describing the system can be written as

$$
\mathcal{F}\{\psi, c\}=\int d \vec{r}\left[\frac{1}{2} K_{\psi}|\vec{\nabla} \psi|^{2}+\frac{1}{2} K_{c}|\vec{\nabla} c|^{2}+f(\psi, c)\right],
$$

where $f(\psi, c)$ is the local bulk free energy density and the gradient terms account for interfaces and inhomogeneities as discussed above. The dynamics of these fields are described by the equations of motion for the nonconserved $\psi$, Eq. (15), and the conserved concentration $c$,

$$
\frac{\partial c}{\partial t}=\Gamma_{c} \nabla^{2} \frac{\delta \mathcal{F}}{\delta c}=\Gamma_{c} \nabla^{2}\left[-K_{c} \nabla^{2} c+\frac{\partial f}{\partial c}\right],
$$

where $\delta \mathcal{F}\{\psi, c\} / \delta c=\mu$ is a chemical potential and $\Gamma_{c}$ is a mobility. The usual additive noise terms, related to the transport coefficients $\Gamma_{c, \psi}$ by fluctuation-dissipation relations $[1,5]$, have been neglected for simplicity. In mean field theory, the equilibrium states of the system with an interface normal to $\hat{y}$ are defined by

$$
K_{\psi} \frac{\partial^{2} \psi}{\partial y^{2}}=\frac{\partial f}{\partial \psi}
$$

and

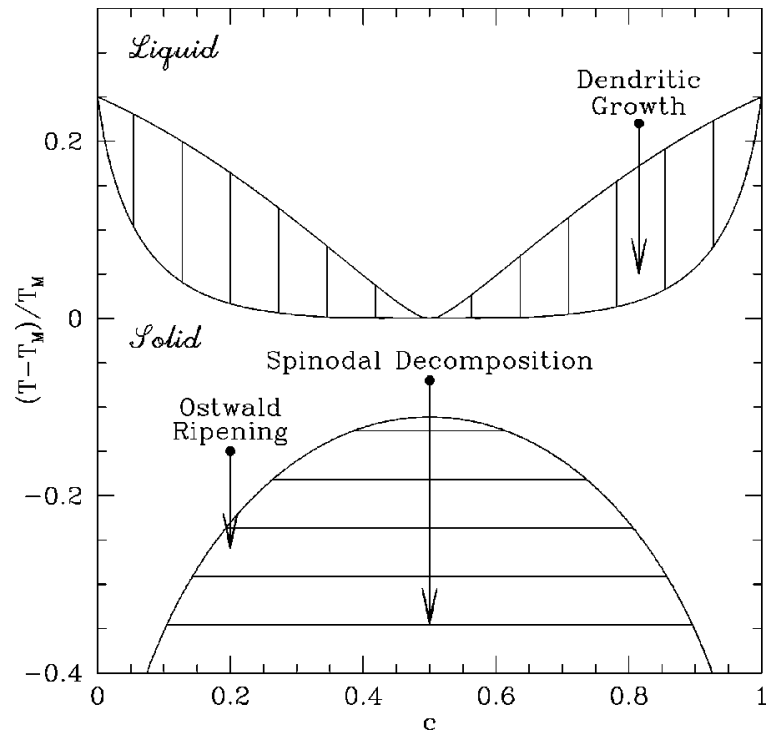

FIG. 1. Mean field phase diagram obtained from the bulk free energy of Eq. (33) for the parameters $\alpha=\beta=1.0$ and $u=0.6$. In this figure the regions containing vertical and horizontal lines are liquid/ solid and solid/solid coexistence regions, respectively.

$$
K_{c} \frac{\partial^{2} c}{\partial y^{2}}=\frac{\partial f}{\partial c}-\mu_{e q}
$$

where $\mu_{e q}$ is the chemical potential of the uniform equilibrium states. Integrating Eq. (32) over $c$ gives the Maxwell's equal area construction rule, $\int_{c_{1}}^{c_{2}} d c\left(\partial f / \partial c-\mu_{e q}\right)=0$, where $c_{1}$ and $c_{2}$ are defined by $\partial f /\left.\partial c\right|_{c_{1}, c_{2}}=\mu_{e q}$.

For a generic $f(c, \psi)$ there may be many possible equilibrium and metastable states contained in this free energy. For illustration, consider the following bulk free energy

$$
\begin{aligned}
f(\psi, c)= & u \frac{T}{T_{M}}[c \ln c+(1-c) \ln (1-c)] \\
& +\left[\alpha \Delta T-\beta\left(c-\frac{1}{2}\right)^{2}\right] \Psi(\psi)-\frac{1}{2} \psi^{2}+\frac{1}{4} \psi^{4} .
\end{aligned}
$$

where $\Psi(\psi) \equiv 2 \psi-4 \psi^{3} / 3+2 \psi^{5} / 5$ and $\Delta T \equiv\left(T-T_{M}\right) / T_{M}$ with $T_{M}$ the melting temperature and the other phenomenological parameters are determined by matching to experimental phase diagrams. If these parameters are chosen as $\alpha$ $=\beta=1.0$ and $u=0.6$, the mean field phase diagram shown in Fig. 1 emerges. As can be seen, this phase diagram contains liquid/solid and solid/solid coexistence regimes. For this symmetric free energy the melting temperature at $c=1 / 2$ is denoted $T_{M}$ and the critical point of the solid/solid coexistence regime is at $(c, T)=\left(1 / 2, T_{c}\right)$ with $T_{c}<T_{M}$. As the parameter $u$ is decreased, $T_{c}$ increases until the solid/solid coexistence region collides with the liquid/solid coexistence regime when $T_{c}>T_{M}$ and a eutectic point is formed at $(c, T)=\left(1 / 2, T_{E}\right)$ as shown in Fig. 2 . 


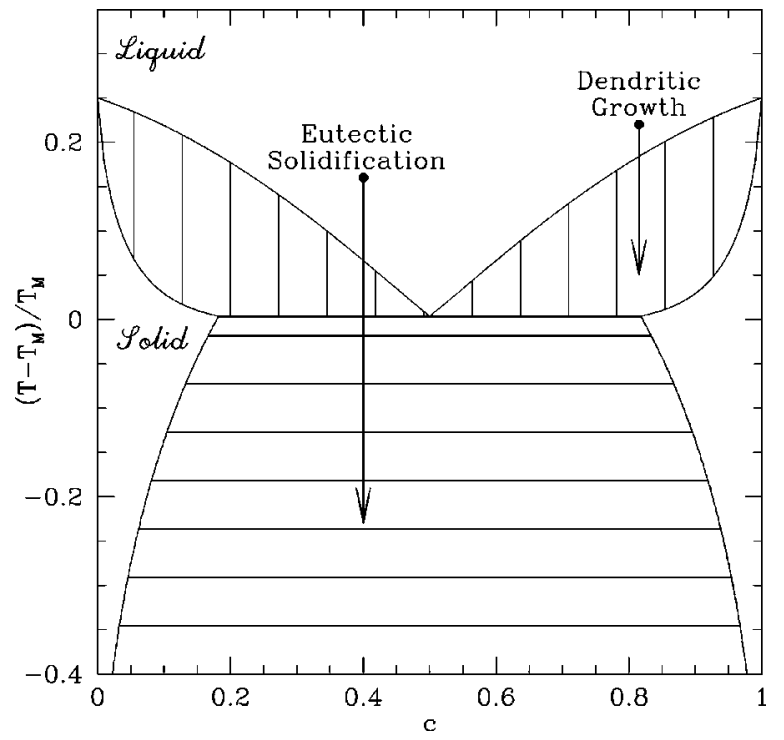

FIG. 2. Mean field phase diagram obtained from bulk free energy given in Eq. (33) for the parameters $\alpha=\beta=1.0$ and $u=0.45$. In this figure the regions containing vertical and horizontal lines are liquid/solid and solid/solid coexistence regions, respectively.

As can be seen from the phase diagrams of Figs. 1 and 2, this simple free energy contains many phases and, in conjunction with appropriate equations of motion, can be used to study a wide variety of phenomena. A number of "quenches" have been highlighted on these diagrams to illustrate several different kinetic processes that may arise. A quench is defined as a rapid change in temperature which takes a system from one region of the phase diagram to another and is often considered instantaneous in theoretical modeling. In the next section the dynamics of an interface separating a stable and a metastable phase is considered. The calculations are done in a general manner to include all the possible quenches shown in Figs. 1 and 2. To construct the relevant sharp-interface equations no reference will be made to the explicit form of the bulk free energy term $f$. It is assumed that $f$ has been chosen merely so that all the phases of interest are well defined. In the remainder of this paper, a detailed implementation of these ideas is presented, making connections to the sharp- or thin-interface limit.

\section{DERIVATION OF INTERFACE EQUATIONS OF MOTION}

The goal of this section is to derive the sharp interface equations for systems described by a free energy functional $\mathcal{F}$ [such as is given by Eqs. (29) and (33)] and the Langevin equations given in Eqs. (15) and (30). These latter equations should be supplemented by additive stochastic noises, $\eta_{\psi}(\vec{r}, t)$ and $\eta_{c}(\vec{r}, t)$ of zero mean and correlations $\left\langle\eta_{\psi}(\vec{r}, t) \eta_{\psi}\left(\vec{r}^{\prime}, t^{\prime}\right)\right\rangle=2 \Gamma_{\psi} T \delta\left(\vec{r}-\vec{r}^{\prime}\right) \delta\left(t-t^{\prime}\right)$ and $\left\langle\eta_{c}(\vec{r}, t) \eta_{c}\left(\vec{r}^{\prime}, t^{\prime}\right)\right\rangle=2 \Gamma_{c} T \nabla^{2} \delta\left(\vec{r}-\vec{r}^{\prime}\right) \delta\left(t-t^{\prime}\right) \quad$ with $\left\langle\eta_{\psi} \eta_{c}\right\rangle=0$ as required by the fluctuation dissipation theorem. With these stochastic noises, the dynamical equations, Eqs. (15) and (30), are the simplest equations which respect the macroscopic conservation laws and also ensure that the

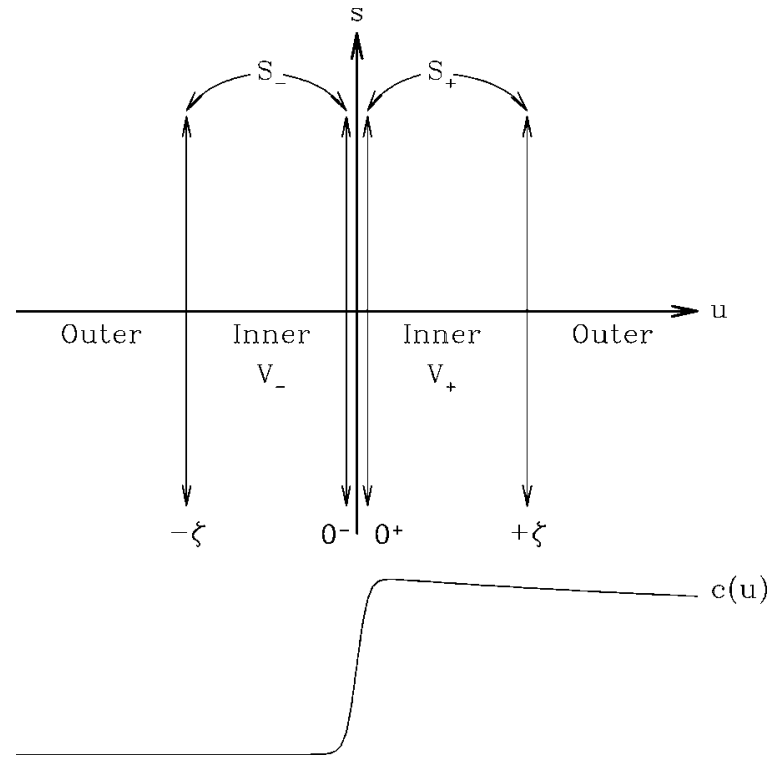

FIG. 3. Illustration of inner and outer regions used in computations.

system evolves towards its ultimate state of thermal and mechanical equilibrium with its external environment.

The asymptotic analysis proceeds by expanding around a planar equilibrium interface described by Eqs. (31) and (32). The interface can be taken out of equilibrium by either gently curving it or by making one of the two bulk phases metastable. In the former case, a gentle curvature is one in which the radius of curvature $1 / \kappa$ is large compared to the interface width or correlation length. Thus one small dimensionless expansion parameter is $\kappa \xi$. In the latter case, the difference between the free energy of the stable and metastable phases causes the interface to propagate into the metastable phase. If the free energy difference is small the propagation velocity $v$ is small. In this context, a small velocity means that the interface moves so slowly that a steady state diffusion field is allowed to form in front of the interface. In other words, the time for the diffusion field to relax when the interface moves a distance $\xi$ should be much smaller than the time $\xi / v$ taken for the interface to move that distance. Since the diffusion time $\tau=\xi^{2} / D$, this leads to another small dimensionless parameter $\xi v / D$ which is known as the interface Péclet number. In the following analysis the interface equations will be obtained to lowest order in both small parameters. Technically the expansion to lowest order in both small parameters can be achieved if they are regarded as the same order in the expansion. In the calculations to follow both parameters will be taken to be $\mathcal{O}(\epsilon)$ with $\epsilon \ll 1$.

The calculations make use of the fact that the fields behave qualitatively differently close to and far from the interface. In the region close to the interface, the fields vary rapidly over distances $\mathcal{O}(\xi)$ while, far from the interface, they vary over distances $\mathcal{O}(\xi / \epsilon)$. If there exists a length scale $\zeta$ such that $1 \ll \zeta / \xi \ll 1 / \epsilon$, then distinct "inner" and "outer" regions can be defined, as shown in Fig. 3, and it is appropriate to solve in both inner and outer regions and match the solutions at the length scale $\zeta$. Formally, the technique re- 
quires an inner expansion near the interface and an outer expansion far from the interface.

\section{A. Inner expansion}

Consider an inner region defined by $-\zeta<u<\zeta$, where $u$ is a coordinate normal to the interface and $1 \ll \zeta / \xi \ll 1 / \epsilon$. The aim is to obtain asymptotic expansions for the solutions to the evolution equations (15) and (30) valid in this inner region. The latter can be written in a compact form as

$$
\frac{1}{\Gamma_{c}} \frac{\partial c}{\partial t}=\nabla^{2} \delta \mu
$$

where

$$
\delta \mu=\mu(\vec{r})-\mu_{e q}=-K_{c} \nabla^{2} c+\frac{\partial f}{\partial c}-\mu_{e q}
$$

The first step is to partition the system into two regions $V_{+}$ and $V_{-}$bounded by surfaces $S_{+}$and $S_{-}$, respectively. The region $V_{+}$is defined by $0<u<\zeta$ and similarly for $V_{-}$. The position of the interface between two bulk regions is defined as $u(\vec{r}, t)=0$. These definitions are purely formal, but to fix ideas, the surface $u(\vec{r}, t)=0$ may be regarded as the surface near which the fields $c, \psi$ vary rapidly over distances $\mathcal{O}(\xi)$. It is then useful to define Green's functions $G^{ \pm}\left(\vec{r}, \vec{r}^{\prime}\right)$ in the regions $-\zeta<u<0 \quad\left(G^{-}\right)$and in $0<u<\zeta \quad\left(G^{+}\right)$obeying

$$
\nabla^{\prime 2} G^{ \pm}\left(\vec{r}, \vec{r}^{\prime}\right)=\delta\left(\vec{r}-\vec{r}^{\prime}\right)
$$

and satisfying the boundary conditions, $G\left(\vec{r}, \vec{r}^{\prime}\right)=0$ at $u$ $=0 \quad$ and $\quad u^{\prime}=0, \quad \partial G\left(\vec{r}, \vec{r}^{\prime}\right) / \partial u=0 \quad$ at $u=$ $\pm \zeta, \quad \partial G\left(\vec{r}, \vec{r}^{\prime}\right) / \partial u^{\prime}=0$ at $u^{\prime}= \pm \zeta$, and periodic in the other directions. Note that both $\vec{r}$ and $\vec{r}^{\prime}$ lie in the same region, $V_{+}$or $V_{-}$.

Multiplying Eq. (34) by $G^{ \pm}$and integrating over $\vec{r}^{\prime}$ $\in V_{ \pm}$gives

$$
\begin{aligned}
\delta \mu(\vec{r})= & \int_{V_{ \pm}} d \vec{r}^{\prime} \frac{G^{ \pm}\left(\vec{r}, \vec{r}^{\prime}\right)}{\Gamma_{c}} \frac{\partial c^{\prime}}{\partial t} \\
& +\oint_{S_{ \pm}} d \vec{S}^{\prime} \cdot\left(\delta \mu^{\prime} \vec{\nabla}^{\prime} G^{ \pm}-G^{ \pm} \vec{\nabla}^{\prime} \delta \mu^{\prime}\right),
\end{aligned}
$$

where $\delta \mu^{\prime}=\delta \mu\left(\vec{r}^{\prime}\right)$ defined as in Eq. (35), $\int_{V_{+}} d \vec{r}^{\prime}$ denotes integration over $V_{+}$defined by $0<u\left(\vec{r}^{\prime}\right)<\zeta$ and $\oint_{S_{+}} d \vec{S}^{\prime}$ denotes integration over the boundaries $S_{+}$enclosing $V_{+}$and similarly for $V_{-}$enclosed by $S_{-}$. The interface dynamics can now be obtained using by the projection method of Kawasaki and Ohta [12]. This is accomplished by multiplying Eq. (37) by $\partial c_{0}^{\text {in }} / \partial u$ where $c_{0}^{\text {in }}(u)$ is determined by the solution of Eqs. (31) and (32) for a planar interface in thermal equilibrium and integrating over $-\zeta<u<+\zeta$ to obtain

$$
\mathcal{B}+\mathcal{S} \equiv \int_{-\zeta}^{\zeta} d u \frac{\partial c_{0}^{\text {in }}}{\partial u} \delta \mu=\int_{-\zeta}^{\zeta} d u \frac{\partial c_{0}^{\text {in }}}{\partial u}\left(\frac{\partial f}{\partial c}-K_{c} \nabla^{2} c-\mu_{e q}\right),
$$

where $\mathcal{B}=\mathcal{B}^{+}+\mathcal{B}^{-}$and $\mathcal{S}=\mathcal{S}^{+}+\mathcal{S}^{-}$with

$$
\begin{gathered}
\mathcal{B}^{ \pm}= \pm \frac{1}{\Gamma_{c}} \int_{0}^{ \pm \zeta} d u \frac{\partial c_{0}^{\text {in }}}{\partial u} \int_{V_{ \pm}} d \vec{r},{ }^{\prime} G^{ \pm}\left(\vec{r}, \vec{r}^{\prime}\right) \frac{\partial c^{\prime}}{\partial t}, \\
\mathcal{S}^{ \pm}= \pm \int_{0}^{ \pm \zeta} d u \frac{\partial c_{0}^{\text {in }}}{\partial u} \oint_{S_{ \pm}} d \vec{S}^{\prime} \cdot\left(\delta \mu^{\prime} \vec{\nabla}^{\prime} G^{ \pm}-G^{ \pm} \vec{\nabla}^{\prime} \delta \mu^{\prime}\right) .
\end{gathered}
$$

An analogous formula for $\psi$ is obtained by multiplying Eq. (15) by $\partial \psi_{0}^{\text {in }} / \partial u$, with $\psi_{0}^{\text {in }}(u)$ the solution of Eqs. (31) and (32), and integrating over $-\zeta<u<\zeta[12]$ to obtain

$$
\frac{1}{\Gamma_{\psi}} \int_{-\zeta}^{\zeta} d u \frac{\partial \psi_{0}^{\text {in }}}{\partial u} \frac{\partial \psi}{\partial t}=\int_{-\zeta}^{\zeta} d u \frac{\partial \psi_{0}^{\text {in }}}{\partial u}\left(K_{\psi} \nabla^{2} \psi-\frac{\partial f}{\partial \psi}\right) .
$$

Each term in the above equations can be systematically expanded in powers of $\epsilon$. In this paper, attention is restricted to the terms $\mathcal{O}(\epsilon)$ as much of the relevant physics is apparent at this order. Going to higher order in $\epsilon$ does not yield any new physical insight but does require considerable bookkeeping skill.

To facilitate the expansion, $c(\vec{r}, t), \quad \psi(\vec{r}, t)$, and the chemical potential $\mu(\vec{r}, t)$ are expanded in a power series in $\epsilon$,

$$
\begin{gathered}
c(\vec{r}, t)=c_{0}^{\mathrm{in}}[u(\vec{r})]+\epsilon \delta c_{1}^{\mathrm{in}}+\epsilon^{2} \delta c_{2}^{\mathrm{in}}+\cdots, \\
\psi(\vec{r}, t)=\psi_{0}^{\mathrm{in}}[u(\vec{r})]+\epsilon \delta \psi_{1}^{\mathrm{in}}+\epsilon^{2} \delta \psi_{2}^{\mathrm{in}}+\cdots, \\
\mu(\vec{r}, t)=\mu_{0}^{\mathrm{in}}[u(\vec{r})]+\epsilon \delta \mu_{1}^{\mathrm{in}}+\epsilon^{2} \delta \mu_{2}^{\mathrm{in}}+\cdots,
\end{gathered}
$$

where the superscript in refers to the inner solution. To expand the Laplacian in powers of $\epsilon$, it is useful to introduce a curvilinear coordinate system with one coordinate $u$ along the local normal to the interface and $(d-1)$ coordinates $\vec{s}$ perpendicular to $u$ and tangent to the interface. For simplicity a two-dimensional system is considered where $s$ is the scalar arclength. Note that the $\mathcal{O}\left(\epsilon^{0}\right)$ terms, $c_{0}^{\text {in }}$ and $\psi_{0}^{\text {in }}$ in Eq. (42), are the equilibrium planar interface solutions of Eqs. (31) and (32).

At this early stage of the calculations it is worth pointing out that the exact position of the interface has not been specified. The choice of the exact interface position is somewhat flexible to within a distance $\xi$ and there is no particular reason for choosing the interface position to be defined by $\psi[u(\vec{r}, t)=0]=0$ as is often done in the literature. Indeed, this particular choice can lead to unreasonable constraints on the free energy for a mapping between the phase-field model and the sharp-interface limit to be possible. As shown by Kawasaki and Ohta [12] for models describing one field (e.g., model A or B in the Halperin and Hohenberg classification scheme [5]) it is convenient to define the interface 
position by setting terms similar to $A_{1}$ to zero. In this work the interface will be chosen to be the Gibbs surface defined so that the excess concentration is equal on both sides of the interface. It will be shown that this is a solvability condition for the matching equations, whose physical interpretation is to ensure continuity of the chemical potential across the interface.

The transformation from Cartesian to curvilinear coordinates (see Appendix A) leads to the formal expansion

$$
\xi^{2} \nabla^{2}=\mathcal{L}_{0}+\epsilon \mathcal{L}_{1}+\epsilon^{2} \mathcal{L}_{2}+\cdots,
$$

where the specific form of $\mathcal{L}_{n}$ depends on the expansion. In the inner region, derivatives of the fields with respect to $u$ are much larger than derivatives with respect to $s$ which are identically zero when the curvature $\kappa$ and the Péclet number vanish. This is taken into account by introducing the dimensionless variables $\bar{u}$ and $\bar{s}$ which are $\mathcal{O}\left(\epsilon^{0}\right)$ by $u=\xi \bar{u}$ and $s=\xi \bar{s} / \epsilon$. As shown in Appendix A, this scaling leads to

$$
\begin{gathered}
\mathcal{L}_{0}=\partial_{\bar{u} u}^{-\bar{u}}, \\
\mathcal{L}_{1}=\bar{\kappa} \partial_{\bar{u}}^{-}, \\
\mathcal{L}_{2}=\partial_{s \bar{s}}-\bar{\kappa}^{2} \bar{u} \partial_{\bar{u}}^{-},
\end{gathered}
$$

where the dimensionless curvature, $\bar{\kappa} \equiv \xi \kappa / \epsilon$, is $\mathcal{O}\left(\epsilon^{0}\right)$.

Lastly, the time derivatives in Eqs. (39) and (41) must be expanded in $\epsilon$. For these calculations, it is convenient to work in the frame in which the interface is stationary so that

$$
\left.\frac{\partial}{\partial t}\right|_{\vec{r}}=\left.\frac{\partial}{\partial t}\right|_{(u, s)}-\vec{v} \cdot \vec{\nabla}
$$

where $\vec{v}$ is the interface velocity which has components normal and tangential to the interface. The time derivative on the right-hand side corresponds to relaxational dynamics not accounted for by motion of the interface. When this operator acts on the fields, $c$ and $\psi$, the tangential component and time derivative are $\mathcal{O}\left(\epsilon^{3}\right)$ and can be dropped [24]. Thus to $\mathcal{O}(\epsilon)$, $\partial /\left.\partial t\right|_{\vec{r}}$ becomes

$$
\left.\frac{\partial}{\partial t}\right|_{\vec{r}}=-\epsilon \frac{v_{1}}{\tau} \frac{\partial}{\partial \bar{u}}+\mathcal{O}\left(\epsilon^{2}\right)+\cdots,
$$

where the normal velocity has been expanded in a power series in $\epsilon$,

$$
v_{\text {normal }} \equiv-\frac{\partial u}{\partial t} \equiv \frac{\xi}{\tau} \sum_{m=1}^{\infty} \epsilon^{m} v_{m}
$$

Using these expansions and expanding $f$ around $c_{0}^{\text {in }}$ and $\psi_{0}^{\text {in }}$ the right-hand sides of Eqs. (38) and (41) become

$$
\begin{aligned}
& \int_{-\zeta}^{\zeta} d u \frac{\partial c_{0}^{\mathrm{in}}}{\partial u}\left[\mu_{0}+\bar{K}_{c} \xi^{2} \nabla^{2} c-\frac{\partial f}{\partial c}\right] \\
& =\int_{-\bar{\zeta}}^{\bar{\zeta}} d \bar{u} \frac{\partial c_{0}^{\mathrm{in}}}{\partial \bar{u}}\left[\left(\mu_{0}+\bar{K}_{c} \mathcal{L}_{0} c_{0}^{\mathrm{in}}-f_{i}^{(1,0)}\right)\right. \\
& \quad+\epsilon\left(\bar{K}_{c}\left(\mathcal{L}_{1} c_{0}^{\mathrm{in}}+\mathcal{L}_{0} \delta c_{1}^{\mathrm{in}}\right)-\delta c_{1}^{\mathrm{in}} f_{i}^{(2,0)}-\delta \psi_{1}^{\mathrm{in}} f_{i}^{(1,1)}\right) \\
& \left.\quad+\mathcal{O}\left(\epsilon^{2}\right)\right]
\end{aligned}
$$

and

$$
\begin{aligned}
\int_{-\zeta}^{\zeta} d u \frac{\partial \psi_{0}^{\text {in }}}{\partial u}\left[\bar{K}_{\psi} \xi^{2} \nabla^{2} \psi-\frac{\partial f}{\partial \psi}\right] \\
\quad=\int_{-\bar{\zeta}}^{\bar{\zeta}} d \bar{u} \frac{\partial \psi_{0}^{\text {in }}}{\partial \bar{u}}\left[\left(\bar{K}_{\psi} \mathcal{L}_{0} \psi_{0}^{\text {in }}-f_{i}^{(0,1)}\right)\right. \\
\quad+\epsilon\left(\bar{K}_{\psi}\left(\mathcal{L}_{1} \psi_{0}^{\text {in }}+\mathcal{L}_{0} \delta \psi_{1}^{\text {in }}\right)\right. \\
\left.\left.\quad-\delta \psi_{1}^{\text {in }} f_{i}^{(0,2)}-\delta c_{1}^{\text {in }} f_{i}^{(1,1)}\right)+\mathcal{O}\left(\epsilon^{2}\right)\right]
\end{aligned}
$$

where $\quad f_{i}^{(n, m)} \equiv \partial^{n+m} f /\left.\partial c^{n} \partial \psi^{m}\right|_{\psi_{0}^{\text {in }}, c_{0}^{\text {in }},} \quad \bar{K}_{c} \equiv K_{c} / \xi^{2}, \quad \bar{K}_{\psi}$ $\equiv K_{\psi} / \xi^{2}$, and $\bar{\zeta}=\zeta / \xi$. Terms of $\mathcal{O}\left(\epsilon^{0}\right)$ vanish by construction. For later use, it is convenient to perform partial integrations on combinations of terms [25]

$$
\begin{aligned}
& \int_{-\bar{\zeta}}^{\bar{\zeta}} d \bar{u} \frac{\partial c_{0}^{\text {in }}}{\partial \bar{u}}\left(\bar{K}_{c} \frac{\partial^{2}}{\partial \bar{u}^{2}}-f_{i}^{(2,0)}\right) \delta c_{1}^{\text {in }} \\
& =\int_{-\bar{\zeta}}^{\bar{\zeta}} d \bar{u} \delta c_{1}^{\text {in }}\left(\bar{K}_{c} \frac{\partial^{2}}{\partial \bar{u}^{2}}-f_{i}^{(2,0)}\right) \frac{\partial c_{0}^{\text {in }}}{\partial \bar{u}} \\
& =\int_{-\bar{\zeta}}^{\bar{\zeta}} d \bar{u} \delta c_{1}^{\text {in }} f_{i}^{(1,1)} \frac{\partial \psi_{0}^{\text {in }}}{\partial \bar{u}}
\end{aligned}
$$

and

$$
\begin{aligned}
& \int_{-\bar{\zeta}}^{\bar{\zeta}} d \bar{u} \frac{\partial \psi_{0}^{\text {in }}}{\partial \bar{u}}\left(\bar{K}_{\psi} \frac{\partial^{2}}{\partial \bar{u}^{2}}-f_{i}^{(0,2)}\right) \delta \psi_{1}^{\text {in }} \\
& =\int_{-\bar{\zeta}}^{\bar{\zeta}} d \bar{u} \delta \psi_{1}^{\text {in }}\left(\bar{K}_{\psi} \frac{\partial^{2}}{\partial \bar{u}^{2}}-f_{i}^{(0,2)}\right) \frac{\partial \psi_{0}^{\text {in }}}{\partial \bar{u}} \\
& =\int_{-\bar{\zeta}}^{\bar{\zeta}} d \bar{u} \delta \psi_{1}^{\text {in }} f_{i}^{(1,1)} \frac{\partial c_{0}^{\text {in }}}{\partial \bar{u}}
\end{aligned}
$$

since derivatives of $c_{0}^{\text {in }}$ and $\psi_{0}^{\text {in }}$ vanish at $\bar{u}= \pm \bar{\zeta}$ in the limit $\bar{\zeta}=\zeta / \xi \gg 1$.

To complete the calculation, the left-hand sides of Eqs. (38) and (41) are expanded to lowest order in $\epsilon$. The expansion for $\psi$ in Eq. (41) is straightforward 


$$
\begin{aligned}
\frac{1}{\Gamma_{\psi}} \int_{-\zeta}^{\zeta} d u \frac{\partial \psi_{0}^{\text {in }}}{\partial u} \frac{\partial \psi}{\partial t} & =-\frac{\epsilon}{\Gamma_{\psi} \tau} \int_{-\bar{\zeta}}^{\bar{\zeta}} d \bar{u} \frac{\partial \psi_{0}^{\text {in }}}{\partial \bar{u}} v_{1} \frac{\partial \psi_{0}^{\text {in }}}{\partial \bar{u}} \\
& =-\epsilon \frac{v_{1} \xi}{\tau} \frac{\sigma_{\psi}}{K_{\psi} \Gamma_{\psi}}+\mathcal{O}\left(\epsilon^{2}\right),
\end{aligned}
$$

where

$$
\sigma_{\psi} \equiv K_{\psi} \int_{-\zeta}^{\zeta} d u\left(\frac{\partial \psi_{0}^{\text {in }}}{\partial u}\right)^{2}
$$

The equivalent expansion for $c$ is more complicated. The algebra is given in Appendix B. Formally, the results of these calculations can be written as

$$
\mathcal{B}+\mathcal{S}=\epsilon\left(\mathcal{B}_{1} / \tau+\mathcal{S}_{1}\right)+\epsilon^{2}\left(\mathcal{B}_{2} / \tau+\mathcal{S}_{2}\right)+\cdots,
$$

where $\mathcal{B}_{n}$ and $\mathcal{S}_{n}$ are given in Appendix B.

Putting all these results together gives the following two equations to $\mathcal{O}(\epsilon)$

$$
\frac{v_{1} \xi}{\tau} \frac{\sigma_{\psi}}{\Gamma_{\psi} K_{\psi}}=-\frac{\bar{\kappa} \sigma_{\psi}}{\xi}-A_{1}
$$

and

$\Delta c \delta \mu_{1}^{\text {in }}(0, s)$

$$
\begin{aligned}
= & -\frac{\sigma_{c} \bar{\kappa}}{\xi}+A_{1}-\frac{v_{1}(s) \xi^{2}}{\tau \Gamma_{c}} \int_{-\bar{\zeta}}^{\bar{\zeta}} d \bar{u}\left[c_{0}^{\text {out }}(\bar{u})-c_{0}^{\text {in }}(\bar{u})\right]^{2} \\
& -\left.\frac{\partial \delta \mu_{1}^{\text {in }}}{\partial \bar{u}}\right|_{-\bar{\zeta}} ^{0} d \bar{u}\left[c_{0}^{\text {out }}(\bar{u})-c_{0}^{\text {in }}(\bar{u})\right] \\
& -\left.\frac{\partial \delta \mu_{1}^{\text {in }}}{\partial \bar{u}}\right|_{\bar{\zeta}} \int_{0}^{\bar{\zeta}} d \bar{u}\left[c_{0}^{\text {out }}(\bar{u})-c_{0}^{\text {in }}(\bar{u})\right]
\end{aligned}
$$

where $\Delta c \equiv c_{0}^{\text {in }}(\bar{\zeta})-c_{0}^{\text {in }}(-\bar{\zeta})$ is the miscibility gap,

$$
\begin{gathered}
c_{0}^{\text {out }}(\bar{u}) \equiv \begin{cases}c_{0}^{\text {in }}(-\bar{\zeta}), & \bar{u}<0 \\
c_{0}^{\text {in }}(+\bar{\zeta}), & \bar{u}>0,\end{cases} \\
\sigma_{c} \equiv K_{c} \int_{-\zeta}^{\zeta} d u\left(\frac{\partial c_{0}^{\text {in }}}{\partial u}\right)^{2},
\end{gathered}
$$

and

$$
A_{1}=\int_{-\bar{\zeta}}^{\bar{\zeta}} d \bar{u}\left(\delta \psi_{1}^{\text {in }} \frac{\partial c_{0}^{\text {in }}}{\partial \bar{u}}-\delta c_{1}^{\text {in }} \frac{\partial \psi_{0}^{\text {in }}}{\partial \bar{u}}\right) f_{i}^{(1,1)}
$$

Equation (55) gives the chemical potential $\mu$ of the inner solution at the interface (i.e., at $\bar{u}=0$ ) which must be matched to the outer solution at $\bar{u}= \pm \bar{\zeta}$. An expression for $\delta \mu_{1}^{\text {in }}( \pm \bar{\zeta})$ can be obtained by expanding Eq. (34) to $\mathcal{O}(\epsilon)$

$$
v_{1} \frac{\partial c_{0}^{\text {in }}}{\partial \bar{u}}=-\frac{\Gamma_{c} \tau}{\xi^{2}} \mathcal{L}_{0} \delta \mu_{1}^{\text {in }} .
$$

Integrating Eq. (59) twice, first from $\bar{u}$ to $\bar{\zeta}$ and then from 0 to $\bar{\zeta}$ yields

$$
\begin{aligned}
\delta \mu_{1}^{\mathrm{in}}(\bar{\zeta}, s)= & \delta \mu_{1}^{\mathrm{in}}(0, s)+\left.\bar{\zeta} \frac{\partial \delta \mu_{1}^{\mathrm{in}}}{\partial \bar{u}}\right|_{\bar{\zeta}}+\frac{v_{1} \xi^{2}}{\tau \Gamma_{c}} \\
& \times \int_{0}^{\bar{\zeta}} d \bar{u}\left[c_{0}^{\text {out }}(\bar{u})-c_{0}^{\text {in }}(\bar{u})\right]
\end{aligned}
$$

and similarly for $\delta \mu_{1}^{\text {in }}(-\bar{\zeta})$. From Eqs. (55) and (60) we obtain

$$
\begin{aligned}
\Delta c \delta \mu_{1}^{\text {in }}( \pm \bar{\zeta}, s)= & -\frac{\sigma_{c} \bar{\kappa}}{\xi}+A_{1} \pm\left.\Delta c \bar{\zeta} \frac{\partial \delta \mu_{1}^{\text {in }}}{\partial \bar{u}}\right|_{ \pm \bar{\zeta}} \\
& -\left.\frac{v_{1} \xi^{2}}{\tau \Gamma_{c}}\right|_{-\bar{\zeta}} ^{\bar{\zeta}} d \bar{u}\left[c_{0}^{\text {out }}(\bar{u})-c_{0}^{\text {in }}(\bar{u})\right]^{2} \\
& -\left.\frac{\partial \delta \mu_{1}^{\text {in }}}{\partial \bar{u}}\right|_{-\bar{\zeta}} \int_{-\bar{\zeta}}^{0} d \bar{u}\left[c_{0}^{\text {out }}(\bar{u})-c_{0}^{\text {in }}(\bar{u})\right] \\
& -\left.\frac{\partial \delta \mu_{1}^{\text {in }}}{\partial \bar{u}}\right|_{\bar{\zeta}} \int_{0}^{\bar{\zeta}} d \bar{u}\left[c_{0}^{\text {out }}(\bar{u})-c_{0}^{\text {in }}(\bar{u})\right] \\
& +\Delta c \frac{v_{1} \xi^{2}}{\tau \Gamma_{c}} \int_{0}^{ \pm \bar{\zeta}} d \bar{u}\left[c_{0}^{\text {out }}(\bar{u})-c_{0}^{\text {in }}(\bar{u})\right] .
\end{aligned}
$$

The integrals in Eq. (61) can be written in a more useful form by noting that $\bar{\zeta} \gg 1$ in the inner region so that $c_{0}^{\text {in }}$ $( \pm \bar{u})=c_{0}^{\text {in }}( \pm \infty)$ for $|\bar{u}| \geqslant \bar{\zeta}$. Equation (61) becomes

$$
\begin{aligned}
\Delta c \delta \mu_{1}^{\text {in }}( \pm \bar{\zeta}, \bar{s})= & -\frac{\sigma_{c} \bar{\kappa}}{\xi}+A_{1} \pm\left.\Delta c \bar{\zeta} \frac{\partial \delta \mu_{1}^{\text {in }}}{\partial \bar{u}}\right|_{ \pm \bar{\zeta}} \\
& -\frac{v_{1}(\bar{s}) \xi^{2}}{\tau \Gamma_{c}} \int_{-\infty}^{+\infty} d \bar{u}\left[c_{0}^{\text {in }}(\bar{u})-c_{0}^{\text {out }}(\bar{u})\right]^{2} \\
& -\left.\frac{\partial \delta \mu_{1}^{\text {in }}}{\partial \bar{u}}\right|_{-\bar{\zeta}} ^{0} d \bar{u}\left[c_{0}^{\text {out }}(\bar{u})-c_{0}^{\text {in }}(\bar{u})\right] \\
& -\left.\frac{\partial \delta \mu_{1}^{\text {in }}}{\partial \bar{u}}\right|_{\bar{\zeta}} ^{+\infty} d \bar{u}\left[c_{0}^{\text {out }}(\bar{u})-c_{0}^{\text {in }}(\bar{u})\right] \\
& +\Delta c \frac{v_{1}(\bar{s}) \xi^{2}}{\tau \Gamma_{c}} \int_{0}^{ \pm \infty} d \bar{u}\left[c_{0}^{\text {out }}(\bar{u})-c_{0}^{\text {in }}(\bar{u})\right] .
\end{aligned}
$$


One last result will be needed and is obtained by integrating Eq. (59) over $-\bar{\zeta}<\bar{u}<\bar{\zeta}$,

$$
v_{1}=-\frac{\tau \Gamma_{c}}{\Delta c \xi^{2}}\left(\left.\frac{\partial \delta \mu_{1}^{\mathrm{in}}}{\partial \bar{u}}\right|_{\bar{\zeta}}-\left.\frac{\partial \delta \mu_{1}^{\mathrm{in}}}{\partial \bar{u}}\right|_{-\bar{\zeta}}\right) .
$$

The solution for $\delta \mu_{1}^{\text {in }}( \pm \bar{\zeta})$ must be matched to the solution in the outer region.

\section{B. Outer expansion}

Far from the interface, the fields $\psi$ and $c$ vary slowly in space and are close to the bulk equilibrium values $\psi_{e q}$ and $c_{e q}$. Variations of the fields in the bulk regions far from the interface take place on length scales $\mathcal{O}(\xi / \epsilon)$ in all directions which implies that suitable dimensionless space and time coordinates are $(\tilde{u}, \tilde{s}, \tilde{t}) \equiv\left(\epsilon u / \xi, \epsilon s / \xi, \epsilon^{2} t / \tau\right)$.

Expanding $\psi(\vec{r}, t)$ about the bulk equilibrium solution $\psi(\vec{r}, t)=\delta \psi^{\text {out }}(\vec{r}, t)+\psi_{e q}$, Eq. (15) becomes

$$
\begin{aligned}
\frac{\partial \delta \psi^{\mathrm{out}}}{\partial t}= & \Gamma_{\psi}\left(K_{\psi} \nabla^{2} \delta \psi^{\mathrm{out}}-\left.\frac{\partial f}{\partial \psi}\right|_{e q}-\left.\frac{\partial^{2} f}{\partial \psi^{2}}\right|_{e q} \delta \psi^{\mathrm{out}}\right. \\
& \left.-\left.\frac{1}{2 !} \frac{\partial^{3} f}{\partial \psi^{3}}\right|_{e q}\left(\delta \psi^{\mathrm{out}}\right)^{2}-\left.\frac{1}{3 !} \frac{\partial^{4} f}{\partial \psi^{4}}\right|_{e q}\left(\delta \psi^{\mathrm{out}}\right)^{3}-\cdots\right) .
\end{aligned}
$$

By definition, $(\partial f / \partial \psi)_{e q}=0$ and, since $\delta \psi^{\text {out }}(\vec{r}, t)=0$ in the limit $\epsilon \rightarrow 0$, Eq. (64) is linear at $\mathcal{O}(\epsilon)$. Furthermore, $\left(\partial^{2} f / \partial \psi^{2}\right)_{e q}>0$ so that $\delta \psi^{\text {out }}(\vec{r}, t)$ vanishes exponentially with time for all wavelengths. Thus $\delta \psi^{\text {out }}(\vec{r}, t)$ is trivial in the outer region and can be ignored. It is convenient to expand $c^{\text {out }}(\vec{r}, t)$ and $\mu^{\text {out }}(\vec{r}, t)$ in the outer region as

$$
\begin{aligned}
c^{\text {out }}(\vec{r}, t) & =c_{0}^{\text {out }}(\vec{r})+\epsilon \delta c_{1}^{\text {out }}(\vec{r}, t)+\cdots, \\
\mu^{\text {out }}(\vec{r}, t) & =\mu_{0}^{\text {out }}(\vec{r})+\epsilon \delta \mu_{1}^{\text {out }}(\vec{r}, t)+\cdots,
\end{aligned}
$$

where $c_{0}^{\text {out }}(\vec{r})$ is given by Eq. (56). At $\mathcal{O}\left(\epsilon^{3}\right)$ in dimensionless variables, in the lab frame

$$
\frac{\partial \delta c_{1}^{\text {out }}}{\partial \tilde{t}}=\frac{\tau \Gamma_{c}}{\xi^{2}} \widetilde{\nabla}^{2} \delta \mu_{1}^{\text {out }},
$$

where $\widetilde{\nabla} \equiv(\xi / \epsilon) \nabla$ is the scaled dimensionless derivative suitable for the outer region. This simplifies to a linear diffusion equation for the chemical potential inside the bulk phases which reads, in dimensional units,

$$
\frac{\partial \mu^{\text {out }}}{\partial t}=D_{c} \nabla^{2} \mu^{\text {out }}
$$

where $D_{c} \equiv \Gamma_{c}\left(\partial \mu^{\text {out }} / \partial c^{\text {out }}\right)_{e q}$ is a diffusion constant. The value of $D_{c}$ depends on the bulk equilibrium phase considered.

\section{Matching and the Gibbs surface}

To solve the diffusion problem of Eq. (67), initial values $\delta \mu_{1}^{\text {out }}(\tilde{u}=0, \tilde{s})$ are required. These are to be obtained by matching to the inner solution $\delta \mu_{1}^{\text {in }}(\bar{u}, \bar{s})$ at $\bar{u}=\bar{\zeta}$. The matching of $\delta \mu_{1}^{\text {in }}(\bar{u}, \bar{s})$ to $\delta \mu_{1}^{\text {out }}(\tilde{u}, \tilde{s})$ and $\partial \delta \mu_{1}^{\text {in }}(\bar{u}, \bar{s}) / \partial \bar{u}$ to $\partial \delta \mu_{1}^{\text {out }} / \partial \tilde{u}$ looks problematical, but it should be remembered that $\mu^{\text {in }}(\vec{r}, t)$ and $\mu^{\text {out }}(\vec{r}, t)$ are exactly the same function expressed in terms of different variables $(\bar{u}, \bar{s})$ and $(\tilde{u}=\epsilon \bar{u}, \tilde{s}$ $=\bar{s})$, respectively, reflecting the scales over which $\mu^{\text {in }}$ and $\mu^{\text {out }}$ vary. Both are expanded as a power series in $\epsilon$ and the functions and their derivatives matched where both expansions are valid. Both are expressed in terms of the same variables $(\bar{u}, \bar{s})$ and matched at $\bar{u}=\bar{\zeta}$. This is spelled out in some detail in the rest of this section.

To $\quad$ obtain $\delta \mu_{1}^{\text {out }}\left(\tilde{u}=0^{ \pm}, \tilde{s}\right) \quad$ from $\quad \delta \mu_{1}^{\text {out }}(\tilde{u}= \pm \tilde{\zeta}, \tilde{s})$ $=\delta \mu_{1}^{\text {in }}(\bar{u}= \pm \bar{\zeta}, \bar{s})$ with $\widetilde{\zeta} \equiv \epsilon \bar{\zeta}$, it is useful to Taylor expand about $\tilde{u}=\widetilde{\zeta}$,

$$
\delta \mu_{1}^{\text {out }}(\tilde{u}, \tilde{s})=\delta \mu_{1}^{\text {out }}( \pm \widetilde{\zeta}, \tilde{s})+\left.(\tilde{u} \mp \widetilde{\zeta}) \frac{\partial \delta \mu_{1}^{\text {out }}}{\partial \tilde{u}}\right|_{ \pm \tilde{\zeta}}+\cdots
$$

In the outer region, $\widetilde{\zeta} \ll 1$ and this expansion is valid at $\tilde{u}$ $=0$

$$
\delta \mu_{1}^{\text {out }}( \pm \widetilde{\zeta}, \widetilde{s})=\delta \mu_{1}^{\text {out }}(0, \tilde{s}) \pm\left.\widetilde{\zeta} \frac{\partial \delta \mu_{1}^{\text {out }}}{\partial \tilde{u}}\right|_{ \pm \tilde{\zeta}}+\cdots
$$

Since $\delta \mu_{1}^{\text {out }}( \pm \widetilde{\zeta})=\delta \mu_{1}^{\text {in }}( \pm \bar{\zeta})$, we can use Eqs. (62) and (69) to obtain

$$
\begin{aligned}
\Delta c \delta \mu_{1}^{\text {out }}(0, \tilde{s}) & =-\frac{\sigma_{c} \bar{\kappa}}{\xi}+A_{1}-\frac{v_{1}(\tilde{s}) \xi^{2}}{\tau \Gamma_{c}} \int_{-\infty}^{+\infty} d \bar{u}\left[c_{0}^{\text {in }}(\bar{u})-c_{0}^{\text {out }}(\bar{u})\right]^{2} \\
& -\left.\frac{\partial \delta \mu_{1}^{\text {in }}}{\partial \bar{u}}\right|_{-\bar{\zeta}} \int_{-\infty}^{0} d \bar{u}\left[c_{0}^{\text {out }}(\bar{u})-c_{0}^{\text {in }}(\bar{u})\right] \\
& -\left.\frac{\partial \delta \mu_{1}^{\text {in }}}{\partial \bar{u}}\right|_{\bar{\zeta}} \int_{0}^{+\infty} d \bar{u}\left[c_{0}^{\text {out }}(\bar{u})-c_{0}^{\text {in }}(\bar{u})\right] \\
& +\Delta c \frac{v_{1}(\tilde{s}) \xi^{2}}{\tau \Gamma_{c}} \int_{0}^{ \pm \infty} d \bar{u}\left[c_{0}^{\text {out }}(\bar{u})-c_{0}^{\text {in }}(\bar{u})\right],
\end{aligned}
$$

which gives the appropriate boundary value of $\delta \mu_{1}^{\text {out }}(0, \widetilde{s})$. The inner solution $\delta \mu_{1}^{\text {in }}(0)$ differs from the outer solution $\delta \mu_{1}^{\text {out }}(0)$ since the matching is done at $\tilde{u}=\widetilde{\zeta}$ and extrapolated linearly to $\tilde{u}=0$ by Eq. (69). The extrapolation and matching of $\delta \mu_{1}^{\text {in }}$ to $\delta \mu_{1}^{\text {out }}$ is illustrated pictorially in Fig. 4.

The right-hand side of Eq. (70) appears to depend on whether the inner and outer solutions are matched at $u=\zeta$ or 


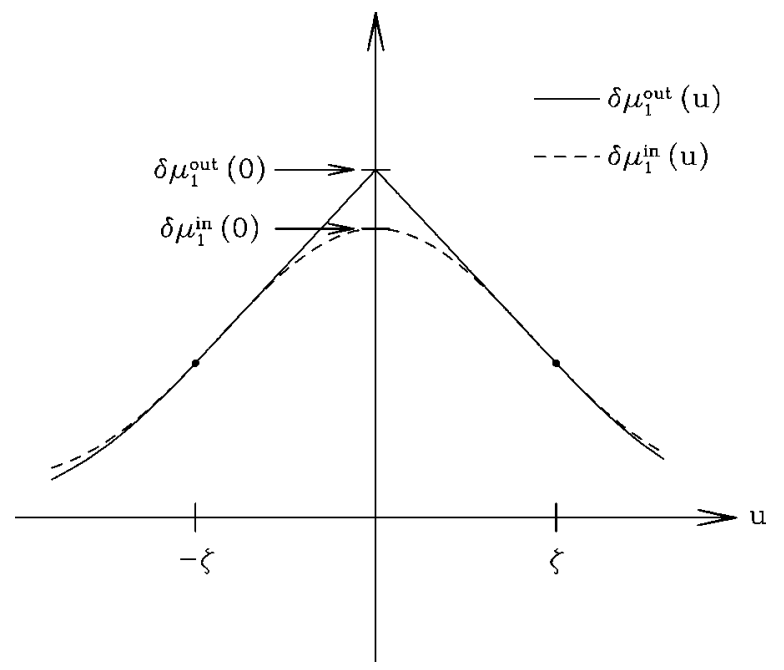

FIG. 4. Matching of $\delta \mu_{1}^{\text {in }}(u)$ (dashed line) with $\delta \mu_{1}^{\text {out }}(u)$ (solid line) at $u= \pm \zeta$.

$u=-\zeta$ because of the last term. This ambiguity is eliminated by defining the interface to be a Gibbs surface at $u=0$ defined by the condition

$$
\int_{-\infty}^{+\infty} d u\left[c_{0}^{\mathrm{out}}(u)-c_{0}^{\mathrm{in}}(u)\right]=0 .
$$

This can always be satisfied by choosing the position of the interface at $u=0$ to be such that Eq. (71) is satisfied. In essence, the interface position is determined by the condition that the excess concentration on one side of the interface is exactly compensated by the deficit on the other, as shown in Fig. 5. This can be regarded as a solvability condition.

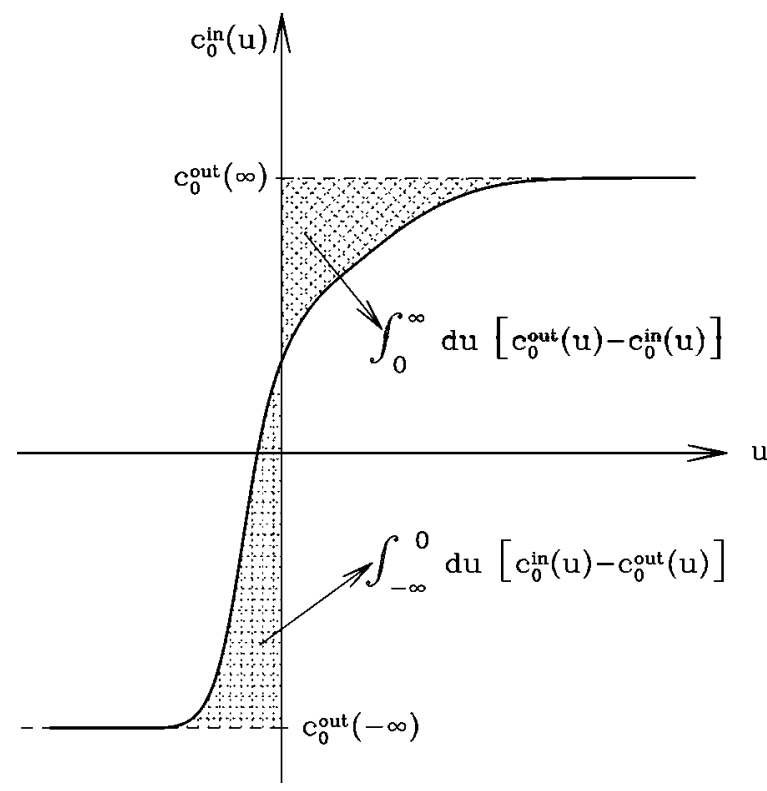

FIG. 5. The Gibbs surface at $u=0$ defined by Eq. (71) which matches the concentration deficit on one side with the concentration excess on the other.
Another result that will be needed is obtained from Eq. (63) by matching the first derivative of the chemical potential. In dimensional units, this reads

$$
v \Delta c=-\Gamma_{c}\left(\left.\frac{\partial \delta \mu^{\mathrm{in}}}{\partial u}\right|_{\zeta}-\left.\frac{\partial \delta \mu^{\mathrm{in}}}{\partial u}\right|_{-\zeta}\right)
$$

Matching derivatives of the inner and outer solutions for $\mu$ and extrapolating back to $u=0^{ \pm}$by Eq. (68), gives the standard result

$$
v \Delta c=-\Gamma_{c}\left(\left.\frac{\partial \mu^{\mathrm{out}}}{\partial u}\right|_{0^{+}}-\left.\frac{\partial \mu^{\mathrm{out}}}{\partial u}\right|_{0^{-}}\right)
$$

since $\mu^{\text {out }}(u)$ is linear for $0<|u| \leqslant \zeta$ from Eq. (68). Finally, combining Eqs. (70), (71), and (73) gives the chemical potential at a moving, curved interface

$$
\Delta c\left[\mu^{\mathrm{out}}(0, s)-\mu_{e q}\right]=-\sigma_{c} \kappa+\mathcal{E}^{2} v+\epsilon A_{1}+\mathcal{O}\left(\epsilon^{2}\right),
$$

where $A_{1}$ is given by Eq. (58) and

$$
\mathcal{E}^{2} \equiv \frac{1}{\Gamma_{c}} \int_{-\infty}^{+\infty} d u\left(\left[c_{0}^{\text {out }}(u)\right]^{2}-\left[c_{0}^{\text {in }}(u)\right]^{2}\right) .
$$

\section{SUMMARY OF RESULTS}

All the results can be combined into a single set of boundary layer equations that can describe many different physical phenomena. Typically the boundary layer equations are written in terms of the concentration, which, in the outer region, is simply related to the chemical potential at $\mathcal{O}(\epsilon)$ by the relationship

$$
\delta \mu=\left.\frac{\partial \mu}{\partial c}\right|_{e q} \delta c .
$$

Combining this result with Eqs. (74) and (54) gives the Gibbs-Thomson relation in dimensional units

$$
\frac{\delta c(0, s)}{\Delta c}=-d_{o} \kappa(s)-\beta v
$$

where $d_{o}$ is the capillary length given by

$$
d_{o}=\frac{\sigma}{(\Delta c)^{2}(\partial \mu / \partial c)_{e q}},
$$

$\sigma \equiv \sigma_{c}+\sigma_{\psi}$ is the total surface tension given by

$$
\sigma=\int_{-\infty}^{\infty} d u\left[K_{\psi}\left(\frac{\partial \psi_{0}^{\text {in }}}{\partial u}\right)^{2}+K_{c}\left(\frac{\partial c_{0}^{\text {in }}}{\partial u}\right)^{2}\right]
$$

and $\beta$ is the coefficient of kinetic undercooling given by

$$
\beta=\frac{1}{(\Delta c)^{2}(\partial \mu / \partial c)_{e q}}\left[\frac{\sigma_{\psi}}{K_{\psi} \Gamma_{\psi}}-\mathcal{E}^{2}\right],
$$


which is obtained by eliminating $A_{1}$ from Eq. (74) by using Eq. (54). Equation (77) provides a boundary condition at the interface for the diffusion equation of Eq. (67) which can be written as

$$
\frac{\partial \delta c}{\partial t}=D_{c} \nabla^{2} \delta c
$$

where

$$
\left.D_{c} \equiv \Gamma_{c} \frac{\partial \mu}{\partial c}\right|_{e q} .
$$

This must be solved in conjunction with Eq. (73) which may be written,

$$
\Delta c v(s)=\left[D_{c} \frac{\partial \delta c}{\partial u}\right]_{0^{-}}-\left[D_{c} \frac{\partial \delta c}{\partial u}\right]_{0^{+}} .
$$

To understand the significance of each term that enters the above equations it is useful to consider some limiting cases. First consider the case in which the concentration field is a constant slightly different from $c_{e q}$, the equilibrium value, $c=c_{e q}+\delta c$. If $\delta \mu$ is the chemical potential difference between the phases defined by the nonconserved field at $u=$ $+\infty$ and $-\infty$, then Eq. (77) reduces to the Allen-Cahn equation in a field,

$$
v=-K_{\psi} \Gamma_{\psi}\left(\kappa+\frac{\delta \mu \Delta c}{\sigma_{\psi}}\right) .
$$

From this point of view the kinetic undercooling can be thought of as simply the relaxation of surface tension in a nonconserved field. Thus the Gibbs-Thomson equation is equivalent to the Allen-Cahn equation in the appropriate limit.

The other simplifying case is when the nonconserved field is a constant as in a pure liquid or solid phase. In this case, the sharp-interface equations remain the same except the coefficients $\sigma$ and $\beta$ become

$$
\sigma=\sigma_{c}
$$

and

$$
\beta=-(\mathcal{E} / \Delta c)^{2} /(\partial \mu / \partial c)_{e q} .
$$

For the conserved case of model $\mathrm{B}, \beta$ is always negative. This term takes into account the lag of the concentration field behind a moving front. When the interface is moving, the interfacial profile cannot instantaneously relax to the correct equilibrium shape $c_{0}^{\text {in }}$. For the conserved field, this correction is roughly as important as dynamic relaxation in the bulk phase, as will be seen in the next section.

\section{LINEAR ANALYSIS}

To illustrate the influence of the various terms that enter the sharp-interface model, it is interesting to study the dynamics of fluctuations around an almost planar interface. To

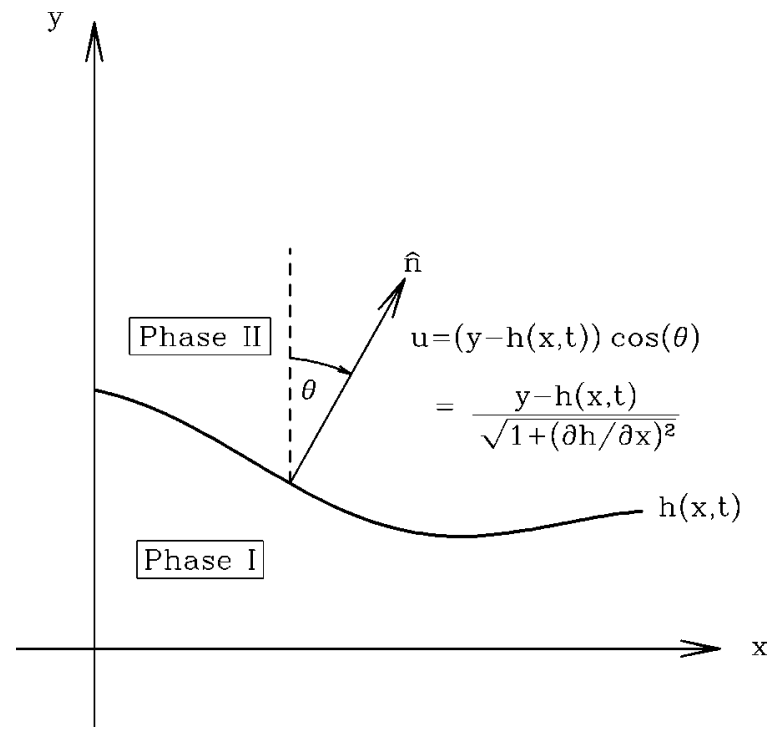

FIG. 6. Interface in Cartesian coordinates.

fix ideas, it is useful to consider an interface separating two phases defined by the equation $y=h(x, t)$ as shown in Fig. 6 . In the calculations to follow it will be assumed that $\vec{\nabla} h(x, t)$ is a small parameter. This is an additional constraint not implicit in the sharp-interface models.

To facilitate the analysis it is worth noting that the normal velocity and curvature can be written in terms of derivatives of $h$ as follows:

$$
v=-\frac{\partial u}{\partial t}=\frac{1}{\gamma} \frac{\partial h}{\partial t}
$$

and

$$
\kappa=-\frac{1}{\gamma^{3}} \frac{\partial^{2} h}{\partial x^{2}},
$$

where $\gamma \equiv \sqrt{1+(\partial h / \partial x)^{2}}$.

\section{A. Nonconserved dynamics}

As discussed in the preceding section, the sharp-interface equations reduce to the Allen-Cahn equation in a field when the conserved field is a constant. When a single valued interface as described above is considered, Eq. (84) reduces to the Kardar-Parisi-Zhang (KPZ) equation [21] in the absence of noise. Substituting Eqs. (87) and (88) into Eq. (84) and linearizing in $h$ gives

$$
\frac{\partial h}{\partial t}=\nu \frac{\partial^{2} h}{\partial x^{2}}+\frac{\lambda}{2}\left(\frac{\partial h}{\partial x}\right)^{2}
$$

where $h \rightarrow h-\lambda t, \nu \equiv \Gamma_{\psi} K_{\psi}$, and $\lambda \equiv-\nu \delta \mu \Delta c / \sigma_{\psi}$. The additive noise term $\eta(x, t)$ in the standard KPZ equation [21] appears when stochastic noise is included in the fundamental Langevin equations.

As a specific example, consider the following free energy: 


$$
\mathcal{F}=\int d \vec{r}\left(-\frac{a}{2} \psi^{2}+\frac{K_{\psi}}{2}|\vec{\nabla} \psi|^{2}+\frac{b}{4} \psi^{4}+d \delta c \psi+\frac{w}{2}(\delta c)^{2}\right)
$$

where $\delta c \equiv c-c_{e q}$ and $c$ is a constant. For this free energy, a planar interface is stationary when $d \rightarrow 0$. This interface is defined by the equations:

$$
\begin{gathered}
\psi_{0}^{\text {in }}=\psi_{e q} \tanh \left(\frac{u}{2 \xi}\right), \\
c_{0}^{\text {in }}=c_{e q}-\frac{d}{w} \psi_{0}^{\text {in }},
\end{gathered}
$$

and

$$
\mu_{e q}=0
$$

where $\xi=\sqrt{K_{\psi} / 2 a}$ and $\psi_{e q}=\sqrt{a / b}$. Thus the miscibility gap, surface tension, and deviations of the chemical potential are given by

$$
\begin{gathered}
\Delta c=-\frac{d}{w} \Delta \psi=-2 \frac{d}{w} \psi_{e q}, \\
\sigma_{\psi}=\frac{2}{3} \frac{K_{\psi} \psi_{e q}^{2}}{\xi},
\end{gathered}
$$

and

$$
\delta \mu=\frac{\partial \mu}{\partial c} \delta c=w \delta c
$$

Thus the coefficient $\lambda$ is given as

$$
\lambda=\Gamma_{\psi} K_{\psi}(d \delta c) \frac{\Delta \psi}{\sigma_{\psi}}=\Gamma_{\psi}(d \delta c) \frac{3}{a} \sqrt{\frac{K_{\psi} b}{2}} .
$$

\section{B. Conserved dynamics}

Now consider an almost planar interface separating two phases of different concentration with the same free energy as occurs, for example, in spinodal decomposition. Since concentration is a conserved field Eqs. (77), (81), and (83) must be solved simultaneously. For simplicity, a two sided model in which $\partial \mu / \partial c$ is the same on both sides of the interface will be considered. This implies that the parameters $d_{o}, \beta$, and $D$ are the same in both phases. It is straightforward to perform the calculations in the more general case but this does not introduce any new physics and is not very illuminating. In the limit $(\partial h / \partial x)^{2} \ll 1$ it is convenient to seek solutions of Eq. (81) of the form

$$
\delta c(u, x)=\delta c(0) e^{(i k x-q|u|+\omega t)},
$$

where $u \approx y-h(x, t)$. For this perturbation it is easy to show that the dimensionless quantities $\bar{\omega} \equiv \omega d_{o}^{2} / D_{c}, \quad \bar{k}$ $\equiv k d_{o}, \quad \bar{q} \equiv q d_{o}$, and the dimensionless kinetic coefficient $\bar{\beta} \equiv \beta D_{c} / d_{o}$ satisfy

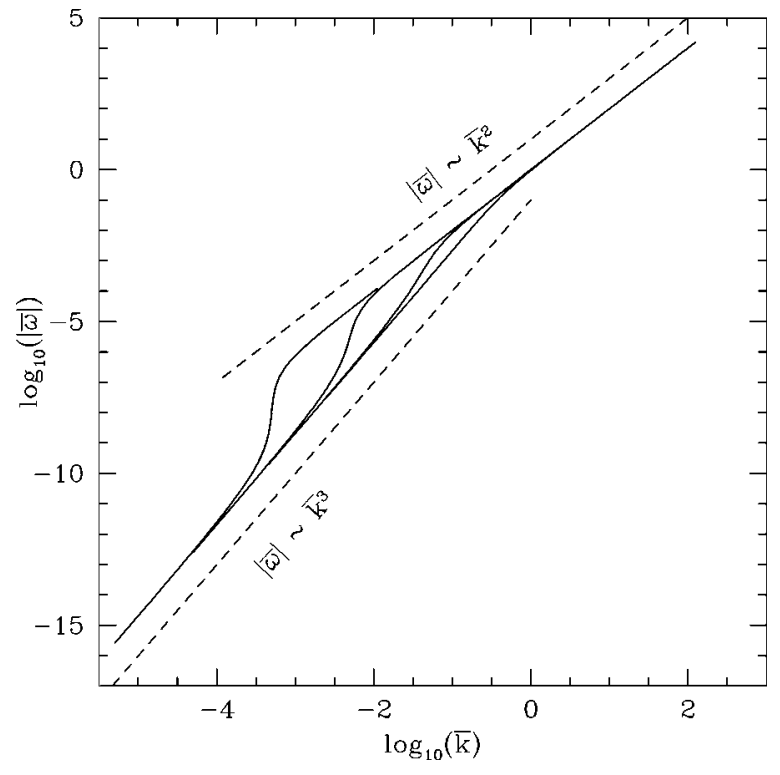

FIG. 7. Dispersion relation for a planar interface separating two conserved phases of equal free energy. The four lines plotted in this figure correspond from right to left $-\bar{\beta}=0,10,100$, and 1000 .

$$
\begin{gathered}
\bar{\omega}=\frac{-2 \bar{q}^{3}}{1-2 \bar{q}(1+|\bar{\beta}|)}, \\
\bar{k}^{2}=\bar{q}^{2}-\bar{\omega} .
\end{gathered}
$$

In the long wavelength limit $(\bar{k} \rightarrow 0), \bar{k} \approx \bar{q}$, and $\bar{\omega} \approx-2 \bar{k}^{3}$, as expected. It is also interesting to note that in the short wavelength limit $(\bar{k} \rightarrow \infty), \bar{q} \rightarrow 1 /(2+2|\bar{\beta}|)$, and $\bar{\omega} \approx-\bar{k}^{2}$. The crossover from the diffusion limited $\omega \sim-k^{2}$ at short wavelength to the asymptotic long wavelength behavior $\omega$ $\sim-k^{3}$ occurs at smaller values of $k$ as the kinetic coefficient $\beta$ becomes more negative. This behavior is sketched in Fig. 7.

This analysis shows that the term $1+|\bar{\beta}|$ in Eq. (97) gives rise to diffusive (i.e., $\omega \sim-k^{2}$ ) behavior and is associated with relaxation in the bulk and in the interface shape. For example, if solutions of the form $e^{i k x-q|u|}$ are sought instead of Eq. (96) this term becomes simply $|\bar{\beta}|$. Thus the " 1 " represents diffusive relaxation in the bulk and the $|\bar{\beta}|$ represents relaxation of the interface shape.

A model commonly used to study spinodal decomposition is known as the Cahn-Hilliard model or model B in the Halperin and Hohenberg classification scheme. The free energy for this model can be written:

$$
\mathcal{F}=\int d \vec{r}\left(-\frac{a}{2} c^{2}+\frac{b}{4} c^{4}+\frac{K_{c}}{2}|\vec{\nabla} c|^{2}\right)
$$

and

$$
\frac{\partial c}{\partial t}=\Gamma_{c} \nabla^{2} \frac{\delta \mathcal{F}}{\delta c}
$$

For this model a stationary planar interface is given by 


$$
c_{0}^{\text {in }}=c_{e q} \tanh \left(\frac{y}{2 \xi}\right) \text {, }
$$

where $\xi=\sqrt{K_{c} / 2 a}$ and $c_{e q}=\sqrt{a / b}$. The coefficients entering the sharp-interface equations are then

$$
\begin{gathered}
d_{o}=\frac{1}{6} \xi \\
\beta=-\frac{\xi}{D_{c}} \rightarrow \bar{\beta}=-6,
\end{gathered}
$$

and

$$
D_{c}=2 \Gamma_{c} a
$$

\section{Nonconserved and conserved dynamics}

Now consider the stability of a stable phase invading a supersaturated liquid phase at constant velocity. This is precisely the situation considered by Langer et al. [2] in the absence of kinetic undercooling and without relaxational kinetics in the bulk phases. It is easy to show that the only solution for a planar front moving at constant velocity which is consistent with the sharp-interface model [i.e., Eqs. (77), $(81)$, and (83)] is

$$
\frac{\delta c_{0}}{\Delta c}=\left\{\begin{array}{l}
\exp \left(-v y^{\prime} / D_{c}\right)-1-\beta v, \quad y^{\prime}>0 \\
-\beta v, \quad y^{\prime}<0
\end{array}\right.
$$

where $v$ is the velocity of the front and $y^{\prime}=y-v t$ is a coordinate in the comoving reference frame.

The stability of this moving front can be determined by studying perturbations about the planar front solution of Eq. (104). We seek solutions of the form

$$
\frac{\delta c}{\Delta c}=\frac{\delta c_{0}}{\Delta c}+ \begin{cases}\delta_{l} \exp \left(i \vec{k} \cdot \vec{x}+\omega t-q y^{\prime}\right), & y^{\prime}>0 \\ \delta_{s} \exp \left(i \vec{k} \cdot \vec{x}+\omega t+q^{\prime} y^{\prime}\right), & y^{\prime}<0\end{cases}
$$

where $q, q^{\prime}>0$, and the position of the perturbed front is at

$$
y^{\prime}=h(\vec{x}, t) \equiv h_{k} \exp (i \vec{k} \cdot \vec{x}+\omega t) .
$$

To linear order in $h_{k}$ and $\delta_{l, s}$, it is straightforward to show that the dimensionless $\bar{\omega}$ and $\bar{k}$ are determined by

$$
\begin{gathered}
\bar{q}^{\prime}=\bar{q}-2 / \bar{l} \\
\bar{\omega}=\frac{2(\bar{q}-2 / \bar{l})[1 / \bar{l}-\bar{q}(\bar{q}-1 / \bar{l})]}{1-2(\bar{q}-1 / \bar{l})(1-\bar{\beta})},
\end{gathered}
$$

and

$$
\bar{k}^{2}=\bar{q}(\bar{q}-2 / \bar{l})-\bar{\omega}
$$

where $\bar{l} \equiv 2 D_{c} / d_{o} v$ and $\bar{\beta} \equiv \beta D_{c} / d_{o}$. The dispersion relation for $\bar{\omega}(\bar{k})$ is plotted in Fig. 8 for $\bar{l}=100$ and several values of $\bar{\beta}$. Note that, in contrast to model B where the kinetic coefficient has a definite $\operatorname{sign} \beta<0$, in model $\mathrm{C}$ it can

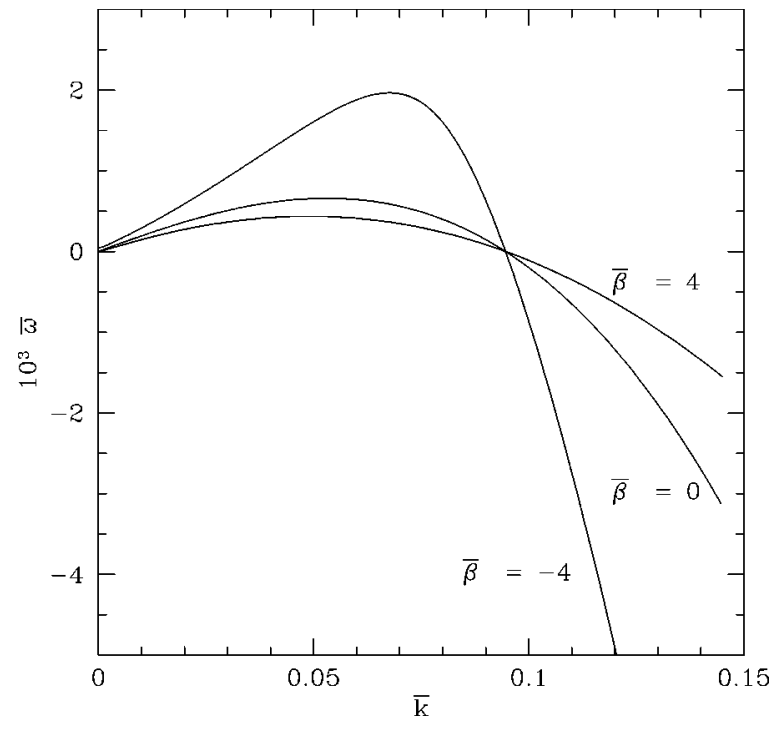

FIG. 8. The linear dispersion relation for the Mullins-Sekerka instability including the kinetic coefficient $\bar{\beta}$, for $\bar{l}=100$

have either sign. Note also that, when $\bar{l} \rightarrow \infty$, Eq. (107) reduces to the result of Eq. (97) for conserved model B dynamics, as it should.

A simplified version of the general system discussed in this paper has been extensively used to study single phase solidification phenomena [10]. The free energy $\mathcal{F}$ can be written as

$$
\mathcal{F}=\int d \vec{r}\left(f(\psi)+\frac{b \lambda}{2} \Phi^{2}+\frac{W^{2}}{2}|\vec{\nabla} \psi|^{2}\right)
$$

where $\Phi \equiv c+h(\psi)$ (called $u$ in Ref. [10]) with

$$
\begin{gathered}
f(\psi)=-\frac{\psi^{2}}{2}+\frac{\psi^{4}}{4}, \\
h(\psi)=\frac{15}{16}\left(\psi-\frac{2}{3} \psi^{3}+\frac{1}{5} \psi^{5}\right) .
\end{gathered}
$$

With this form of $f(\psi)$ the interface width is $W$. The dynamical evolution is governed by Langevin equations for the conserved field $c$ and the nonconserved $\psi$ which, in the noiseless limit, are

$$
\begin{gathered}
\frac{\partial \psi}{\partial t}=-\frac{1}{\tau} \frac{\delta \mathcal{F}}{\delta \psi}, \\
\frac{\partial c}{\partial t}=\frac{D_{c}}{b \lambda} \nabla^{2} \frac{\delta \mathcal{F}}{\delta c} .
\end{gathered}
$$

A stationary planar interface is given by

$$
\begin{gathered}
\psi_{0}^{\text {in }}(u)=\tanh \left(\frac{u}{W \sqrt{2}}\right), \\
c_{0}^{\text {in }}(u)=-h\left(\psi_{0}^{\text {in }}\right) .
\end{gathered}
$$


For an interface with curvature $\kappa$ propagating with velocity $v$, it is tedious but straightforward to use the Gibbs-Thomson relation of Eq. (77) to find

$$
\begin{aligned}
\Phi(0)= & -\left(\frac{W}{\lambda}\right)\left(\frac{5}{4 \sqrt{2}}\right) \kappa-\left[\left(\frac{5}{4 \sqrt{2}}\right)\left(\frac{\tau}{W \lambda}\right)-\left(\frac{209 \sqrt{2}}{840}\right)\right. \\
& \left.\times\left(\frac{W}{D}\right)\right] v .
\end{aligned}
$$

The term inside the square brackets of Eq. (112) is proportional to the kinetic coefficient $\beta$ and contains the sum of the positive model A contribution and the negative model B part. In principle the kinetic coefficient $\beta$ can be of either sign in solidification processes while it must be negative for any process described by model $\mathrm{B}$, such as phase separation in binary alloys.

It is also trivial to show that

$$
\begin{gathered}
v=-D_{c}\left(\left.\frac{\partial c}{\partial u}\right|_{+}-\left.\frac{\partial c}{\partial u}\right|_{-}\right), \\
\frac{\partial c}{\partial t}=D_{c} \nabla^{2} c .
\end{gathered}
$$

These results are identical to those found numerically by others [10].

\section{SUMMARY}

The use of continuum phase-field models to describe phenomena involving the motion of well-defined sharp interfaces is discussed. The phase-field models involve interfaces which are diffuse on a length scale of $\xi$. Considering a general class of phase-field models, it is shown how equations describing the sharp-interface limit are obtained when $\xi \kappa$ $\ll 1$ and $\xi v / D \ll 1$. It is also shown that the Allen-Cahn equation is a special case of the Gibbs-Thomson relation.

In particular, it should be emphasized that these calculations are independent of the specific form of the free energy functional, provided $\mathcal{F}$ describes well-defined phases. Furthermore, the calculations are universal: a large class of free energies give rise to sharp-interface equations which differ only in the values of parameters but are of the same functional form. To realize this goal, it is essential that the "sharp-interface limit" involves an interface of finite width, $\xi$. Expansions involving a zero width interface require a delicate and unphysical tuning of parameters in the free energy for thermodynamic consistency. In this work, the small parameters $\kappa \xi$ and $v \xi / D$ vanish when the curvature $\kappa$ and the inverse diffusion length $v / D$ go to zero for a finite interface thickness $\xi$. Thus delicate tuning is not required for thermodynamic consistency in our approach which is based on the fundamental principles of statistical mechanics.

This work opens the way to construct physically consistent sharp-interface descriptions of more complicated multiple phase systems such as a solid in contact with a fluid which can support flows. This will involve mode coupling

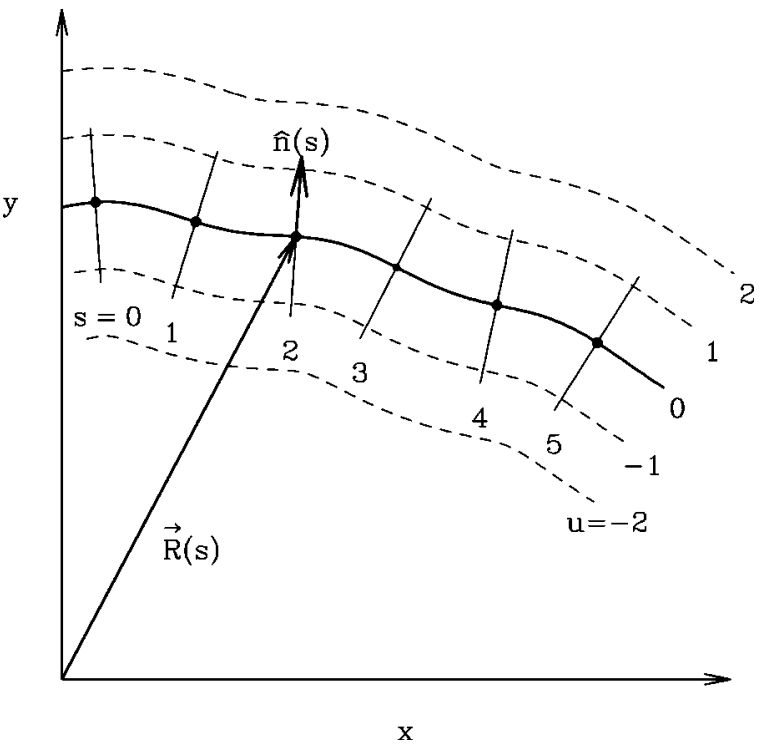

FIG. 9. Curvilinear coordinates.

terms in the dynamical equations. Once such Langevin equations are constructed, there should be no conceptual difficulty in deriving the corresponding interface equations.

\section{ACKNOWLEDGMENTS}

This work was supported by the Natural Sciences and Engineering Research Council of Canada (M.G.), le Fonds pour la Formation de Chercheurs et l'Aide à la Recherche du Québec (M.G.), Research Corporation Grant No. CC4787 (K.R.E.), NSF-DMR Grant No. 0076054 (K.R.E.), and NASA Grant No. NAG3-1929 (J.M.K.).

\section{APPENDIX A: CURVILINEAR COORDINATES}

The curvilinear coordinates $(u, s)$ used in the text are related to the Cartesian coordinates in the following manner

$$
\vec{r}=\vec{R}(s)+u \hat{n}(s),
$$

where $\vec{R}$ is the position of the interface and $\hat{n}(s)$ is the normal vector [see Fig. 9]. The metric tensor $g_{\alpha \beta}$ of the transformation from Cartesian to curvilinear coordinates is

$$
g=\left(\begin{array}{cc}
1 & 0 \\
0 & (1+u \kappa)^{2}
\end{array}\right),
$$

where $\kappa=\partial \theta / \partial s$ is the curvature with $\theta$ the angle between the $x$ axis and the tangent to the curve. The Laplacian in $(u, s)$ is then obtained in the usual manner

$$
\begin{aligned}
\nabla^{2} & =\sum_{\alpha, \beta} \frac{1}{\sqrt{|g|}} \frac{\partial}{\partial x^{\alpha}} \sqrt{|g|} g^{\alpha \beta} \frac{\partial}{\partial x^{\beta}} \\
& =\frac{\partial^{2}}{\partial u^{2}}+\frac{\kappa}{1+u \kappa} \frac{\partial}{\partial u}+\frac{1}{(1+u \kappa)^{2}} \frac{\partial^{2}}{\partial s^{2}}-\frac{u \kappa_{s}}{(1+u \kappa)^{3}} \frac{\partial}{\partial s},
\end{aligned}
$$


where $x^{1}=u, x^{2}=s$, and $g^{\alpha \beta}$ are the components of the inverse of the matrix $g$ and $\kappa_{s} \equiv \partial \kappa / \partial s$.

In the inner region the fields vary much more rapidly in the $u$ direction than the $s$ direction. The coordinates $(u, s)$ are rescaled in dimensionless units as $(\bar{u}, \bar{s}) \equiv(u / \xi, \epsilon s / \xi)$. The dimensionless curvature $\bar{\kappa}=\xi \kappa / \epsilon$ and $\bar{\kappa}_{s}^{-}=\xi^{2} \kappa_{s} / \epsilon^{2}$. In terms of these dimensionless variables, the Laplacian becomes

$$
\begin{aligned}
\xi^{2} \nabla^{2}= & \frac{\partial^{2}}{\partial \bar{u}^{2}}+\frac{\epsilon \bar{\kappa}}{1+\epsilon \bar{u} \bar{\kappa}} \frac{\partial}{\partial \bar{u}}+\frac{\epsilon^{2}}{(1+\epsilon \bar{u} \bar{\kappa})^{2}} \frac{\partial^{2}}{\partial \bar{s}^{2}} \\
& -\frac{\epsilon^{3} \bar{u} \bar{\kappa}-\bar{s}}{(1+\epsilon \bar{u} \bar{\kappa})^{3}} \frac{\partial}{\partial \bar{s}} \\
= & \frac{\partial^{2}}{\partial \bar{u}^{2}}+\bar{\kappa}\left(\epsilon \sum_{n=0}(-\epsilon \bar{u} \bar{\kappa})^{n}\right) \frac{\partial}{\partial \bar{u}}+\left(\epsilon^{2} \sum_{n=0}(n+1)\right. \\
& \left.\times(-\epsilon \bar{u} \bar{\kappa})^{n}\right) \frac{\partial^{2}}{\partial \bar{s}^{2}}-\frac{\bar{u} \bar{\kappa}_{s}^{-}}{2}\left(\epsilon^{3} \sum_{n=0}(n+1)(n+2)\right. \\
& \left.\times(-\epsilon \bar{u} \bar{\kappa})^{n}\right) \frac{\partial}{\partial \bar{s}} \\
= & \mathcal{L}_{0}+\epsilon \mathcal{L}_{1}+\epsilon^{2} \mathcal{L}_{2}+\epsilon^{3} \mathcal{L}_{3}+\cdots,
\end{aligned}
$$

where

$$
\begin{gathered}
\mathcal{L}_{0}=\partial^{2} / \partial \bar{u}^{2}, \\
\mathcal{L}_{1}=\bar{\kappa} \partial / \partial \bar{u}, \\
\mathcal{L}_{2}=\partial^{2} / \partial \bar{s}^{2}-\bar{\kappa}^{2} \bar{u} \partial / \partial \bar{u},
\end{gathered}
$$

and

$$
\mathcal{L}_{3}=-2 \bar{u} \bar{\kappa} \partial^{2} / \partial \bar{s}^{2}+\bar{\kappa}^{3} \bar{u}^{2} \partial / \partial \bar{u}-\bar{u} \bar{\kappa}_{\bar{s}}^{-} \partial / \partial \bar{s} .
$$

\section{APPENDIX B: GREEN'S FUNCTIONS}

It will also be useful to develop an expansion for the inverse of the Laplacian or Green's function. The Green's function of interest is defined by

$$
\nabla_{\vec{r}}^{2} G\left(\vec{r}, \vec{r}^{\prime}\right)=\delta\left(\vec{r}-\vec{r}^{\prime}\right) .
$$

An expansion of the Green's function can be obtained in a straightforward manner. Let $G\left(\vec{r}, \vec{r}^{\prime}\right)=G_{0}\left(\bar{u}, \bar{s} ; \bar{u}^{\prime}, \bar{s}^{\prime}\right)$ $+\epsilon G_{1}\left(\bar{u}, \bar{s} ; \bar{u}^{\prime}, \bar{s}^{\prime}\right)+\cdots$ where

$$
\begin{gathered}
\mathcal{L}_{0} G_{0}=0, \\
\mathcal{L}_{0} G_{1}+\mathcal{L}_{1} G_{0}=\delta\left(\bar{u}-\bar{u}^{\prime}\right) \delta\left(\bar{s}-\bar{s}^{\prime}\right), \\
\mathcal{L}_{0} G_{2}+\mathcal{L}_{1} G_{1}+\mathcal{L}_{2} G_{0}=0,
\end{gathered}
$$

and so on. The solution for $G_{0}$ is $G_{0}=0$, so that the lowest order solution for $G$ is $G_{1}$, which satisfies the equation

$$
\frac{\partial^{2}}{\partial \bar{u}^{2}} G_{1}\left(\bar{u}, \bar{s} ; \bar{u}^{\prime}, \bar{s}^{\prime}\right)=\delta\left(\bar{u}-\bar{u}^{\prime}\right) \delta\left(\bar{s}-\bar{s}^{\prime}\right)
$$

which has the solution

$$
\begin{aligned}
& G_{1}^{-}\left(\bar{u}, \bar{s}_{i} ; \bar{u}^{\prime}, \bar{s}^{\prime}\right)=\left\{\begin{array}{lc}
+\bar{u} \delta\left(\bar{s}-\bar{s}^{\prime}\right), & -\zeta<\bar{u}^{\prime}<\bar{u}<0 \\
+\bar{u}^{\prime} \delta\left(\bar{s}-\bar{s}^{\prime}\right), & -\zeta<\bar{u}<\bar{u}^{\prime}<0,
\end{array}\right. \\
& G_{1}^{+}\left(\bar{u}, \bar{s} ; \bar{u}^{\prime}, \bar{s}^{\prime}\right)=\left\{\begin{array}{lc}
-\bar{u}^{\prime} \delta\left(\bar{s}-\bar{s}^{\prime}\right), & 0<\bar{u}^{\prime}<\bar{u}<+\zeta \\
-\bar{u} \delta\left(\bar{s}-\bar{s}^{\prime}\right), & 0<\bar{u}<\bar{u}^{\prime}<+\zeta .
\end{array}\right.
\end{aligned}
$$

The surface terms $\mathcal{S}^{ \pm}$of Eq. (40) can be expanded as

$$
\begin{aligned}
\mathcal{S}^{ \pm}= & \pm \int_{0}^{ \pm \bar{\zeta}} d \bar{u} \frac{\partial c_{0}^{\mathrm{in}}}{\partial \bar{u}} \oint_{B} d \bar{s}^{\prime}\left[\delta \mu\left(\bar{u}^{\prime}, \bar{s}^{\prime}\right) \frac{\partial G^{ \pm}}{\partial \bar{u}^{\prime}}\right. \\
& \left.-G^{ \pm} \frac{\partial \delta \mu\left(\bar{u}^{\prime}, \bar{s}^{\prime}\right)}{\partial \bar{u}^{\prime}}\right]\left.\right|_{B} \\
= & \pm \int_{0}^{ \pm \bar{\zeta}} d \bar{u} \frac{\partial c_{0}^{\mathrm{in}}}{\partial \bar{u}} \oint_{B} d \bar{s}^{\prime} \sum_{n=1} \sum_{m=0} \epsilon^{n+m-1} \\
& \times\left[\delta \mu_{n}\left(\bar{u}^{\prime}, \bar{s}^{\prime}\right) \frac{\partial G_{m}^{ \pm}\left(\bar{u}, \bar{s}_{\bar{s}} ; \bar{u}^{\prime}, \bar{s}^{\prime}\right)}{\partial \bar{u}^{\prime}}\right. \\
& \left.-G_{m}^{ \pm}\left(\bar{u}, \bar{s}_{\bar{s}} ; \bar{u}^{\prime}, \bar{s}^{\prime}\right) \frac{\left.\partial \delta \mu_{n}\left(\bar{u}^{\prime}, \bar{s}^{\prime}\right)\right]}{\partial \bar{u}^{\prime}}\right]\left.\right|_{B} \\
= & \epsilon \mathcal{S}_{1}^{ \pm}+\epsilon^{2} \mathcal{S}_{2}^{ \pm}+\cdots,
\end{aligned}
$$

where

$$
\begin{gathered}
\mathcal{S}_{1}^{ \pm}= \pm\left.\int_{0}^{ \pm \bar{\zeta}} d \overline{\bar{u}} \frac{\partial c_{0}^{\text {in }}}{\partial \bar{u}} \oint_{B} d \bar{s}^{\prime}\left(\delta \mu_{1} \frac{\partial G_{1}^{ \pm}}{\partial \bar{u}^{\prime}}-G_{1}^{ \pm} \frac{\partial \delta \mu_{1}}{\partial \bar{u}^{\prime}}\right)\right|_{B}, \\
\mathcal{S}_{2}^{ \pm}= \pm \int_{0}^{ \pm \bar{\zeta}} d \overline{\bar{u}} \frac{\partial c_{0}^{\text {in }}}{\partial \bar{u}} \oint_{B} d \bar{s}^{\prime}\left[\delta \mu_{2} \frac{\partial G_{1}^{ \pm}}{\partial \bar{u}^{\prime}}+\delta \mu_{1} \frac{\partial G_{2}^{ \pm}}{\partial \bar{u}^{\prime}}\right. \\
\left.-G_{2}^{ \pm} \frac{\partial \delta \mu_{1}}{\partial \bar{u}^{\prime}}-G_{1}^{ \pm} \frac{\partial \delta \mu_{2}}{\partial \bar{u}^{\prime}}\right]\left.\right|_{B},
\end{gathered}
$$

where the subscript $B$ indicates that the integrands are evaluated on the boundary at $\bar{u}^{\prime}=0^{ \pm}$and at $\bar{u}^{\prime}= \pm \bar{\zeta}$.

The $\mathcal{O}(\epsilon)$ surface contribution becomes

$$
\begin{aligned}
\mathcal{S}_{1}= & \mathcal{S}_{1}^{-}+\mathcal{S}_{1}^{+} \\
= & \int_{-\bar{\zeta}}^{0} d \bar{u} \frac{\partial c_{0}^{\mathrm{in}}}{\partial \bar{u}}\left(\delta \mu_{1}^{\mathrm{in}}(0, \bar{s})+\left.\bar{u} \frac{\partial \delta \mu_{1}^{\mathrm{in}}\left(\bar{u}^{\prime}, \bar{s}\right)}{\partial \bar{u}^{\prime}}\right|_{-\bar{\zeta}}\right) \\
& +\int_{0}^{\bar{\zeta}} d \bar{u} \frac{\partial c_{0}^{\mathrm{in}}}{\partial \bar{u}}\left(\left.\bar{u} \frac{\partial \delta \mu_{1}^{\mathrm{in}}\left(\bar{u}^{\prime}, \bar{s}\right)}{\partial \bar{u}^{\prime}}\right|_{\bar{\zeta}}+\delta \mu_{1}^{\mathrm{in}}(0, \bar{s})\right)
\end{aligned}
$$




$$
\begin{aligned}
= & \delta \mu_{1}^{\mathrm{in}}(0, \bar{s})+\left.\frac{\partial \delta \mu_{1}^{\mathrm{in}}}{\partial \bar{u}^{\prime}}\right|_{-\bar{\zeta}} \int_{-\bar{\zeta}}^{0} d \bar{u}\left[c_{0}^{\mathrm{in}}(-\bar{\zeta})-c_{0}^{\mathrm{in}}(\bar{u})\right] \\
& +\left.\frac{\partial \delta \mu_{1}^{\mathrm{in}}}{\partial \bar{u}^{\prime}}\right|_{\bar{\zeta}} \int_{0}^{\bar{\zeta}} d \bar{u}\left[c_{0}^{\mathrm{in}}(+\bar{\zeta})-c_{0}^{\mathrm{in}}(\bar{u})\right] .
\end{aligned}
$$

To evaluate the bulk contribution $\mathcal{B}$, Eq. (39) is expanded in powers of $\epsilon$. Equation (39) reads

$$
\mathcal{B}^{ \pm}= \pm \frac{1}{\Gamma_{c}} \int_{0}^{ \pm \zeta} d u \frac{\partial c_{0}^{\text {in }}}{\partial u} \int_{V_{ \pm}} d \vec{r}^{\prime} G^{ \pm}\left(\vec{r}, \vec{r}^{\prime}\right) \frac{\partial c^{\text {in }}\left(\vec{r}^{\prime}, t\right)}{\partial t} .
$$

We note that $\partial c^{\text {in }}\left(\vec{r}^{\prime}, t\right) / \partial t \equiv v \partial c^{\text {in }} / \partial u^{\prime}=\mathcal{O}(\epsilon)$ since the normal interface velocity $v=\epsilon v_{1}+\cdots, c^{\text {in }}=c_{0}^{\text {in }}+\epsilon \delta c_{1}^{\text {in }}+\cdots$, and $G^{ \pm}=\epsilon G_{1}^{ \pm}+\cdots$. Naive power counting seems to imply that $\mathcal{B}^{ \pm}=\mathcal{O}\left(\epsilon^{2}\right)$, but changing variables $\vec{r}^{\prime} \rightarrow \bar{u}^{\prime}, \bar{s}^{\prime}$ yields

$$
\begin{aligned}
\mathcal{B}^{ \pm}= & \pm \frac{\xi^{2}}{\tau \Gamma_{c}} \int_{0}^{ \pm \bar{\zeta}} d \bar{u} \int_{V_{ \pm}} \frac{d \bar{u}^{\prime} d \bar{s}^{\prime}}{1+\bar{u}^{\prime} \epsilon \bar{\kappa}\left(\bar{s}^{\prime}\right)} \sum_{n=1} \sum_{m=1} \epsilon^{n+m-1} \\
& \times v_{n} G_{m}^{ \pm}\left(\bar{u}, \bar{s}_{\bar{u}} ; \bar{u}^{\prime}, \bar{s}^{\prime}\right) \frac{\partial c_{0}^{\mathrm{in}}}{\partial \bar{u}} \frac{\partial}{\partial \bar{u}^{\prime}}\left(c_{0}^{\mathrm{in}}+\epsilon \delta c_{1}^{\mathrm{in}}+\cdots\right) \\
= & \epsilon \mathcal{B}_{1}^{ \pm}+\epsilon^{2} \mathcal{B}_{2}^{ \pm}+\cdots,
\end{aligned}
$$

where

$$
\begin{aligned}
\mathcal{B}_{1}^{ \pm}= & \pm \frac{\xi^{2}}{\tau \Gamma_{c}} \int_{0}^{ \pm \bar{\zeta}} d \bar{u} \int_{V_{ \pm}} d \bar{u}^{\prime} d \bar{s}^{\prime} v_{1} G_{1}^{ \pm} \frac{\partial c_{0}^{\text {in }}}{\partial \bar{u}} \frac{\partial c_{0}^{\text {in }}}{\partial \bar{u}^{\prime}}, \\
\mathcal{B}_{2}^{ \pm}= & \pm \frac{\xi^{2}}{\tau \Gamma_{c}} \int_{0}^{ \pm \bar{\zeta}} d \bar{u} \int_{V_{ \pm}} d \bar{u}^{\prime} d \bar{s}^{\prime}\left[\left(v_{2} G_{1}^{ \pm}+v_{1} G_{2}^{ \pm}\right.\right. \\
& \left.\left.-v_{1} \bar{\kappa} \bar{u}^{\prime} G_{1}^{ \pm}\right) \frac{\partial c_{0}^{\text {in }}}{\partial \bar{u}} \frac{\partial c_{0}^{\text {in }}}{\partial \bar{u}^{\prime}}+v_{1} G_{1}^{ \pm} \frac{\partial c_{0}^{\text {in }}}{\partial \bar{u}} \frac{\partial \delta c_{1}^{\text {in }}}{\partial \bar{u}^{\prime}}\right] .
\end{aligned}
$$

For the Green's function introduced above, $\mathcal{B}_{1}$ becomes

$$
\mathcal{B}_{1}=\mathcal{B}_{1}^{-}+\mathcal{B}_{1}^{+}=-\frac{\xi^{2} v_{1}(\bar{s})}{\tau \Gamma_{c}} \int_{-\bar{\zeta}}^{\bar{\zeta}} d \bar{u}\left[c_{0}^{\text {in }}(\bar{u})-c_{0}^{\text {out }}(\bar{u})\right]^{2} .
$$

\section{APPENDIX C: TWO-SIDED MOBILITY}

In this Appendix the sharp-interface equations are outlined for a mobility that takes on a constant value in each phase. There is the question of consistency of such a theory in the presence of stochastic noises in the underlying Langevin equations which we do not attempt to answer. We consider the system in the unphysical limit of zero noise. For convenience, the mobility in the phase in the region $u$ $<0 \quad(u>0)$ is denoted $\Gamma_{c}^{-}\left(\Gamma_{c}^{+}\right)$.

When the mobility $\Gamma_{c}$ is different in the two phases, the equation of motion for the concentration $c$ becomes

$$
\frac{\partial c}{\partial t}=\vec{\nabla}\left(\Gamma_{c} \cdot \vec{\nabla} \frac{\delta \mathcal{F}}{\delta c}\right)=\vec{\nabla}\left(\Gamma_{c} \cdot \vec{\nabla} \delta \mu\right)=\Gamma_{c} \nabla^{2} \delta \mu+\left(\vec{\nabla} \Gamma_{c}\right) \cdot \vec{\nabla} \delta \mu .
$$

The procedure outlined in the main text gives

$$
\begin{aligned}
\int_{V_{ \pm}} d \vec{r}^{\prime} \frac{G^{ \pm}\left(\vec{r}, \vec{r}^{\prime}\right)}{\Gamma_{c}^{\prime}} \frac{\partial c^{\prime}}{\partial t}= & \delta \mu-\int_{S_{ \pm}} d \vec{S}^{\prime} \cdot\left(\delta \mu^{\prime} \vec{\nabla}^{\prime} G^{ \pm}\right. \\
& \left.-G^{ \pm} \vec{\nabla}^{\prime} \delta \mu^{\prime}\right) \\
& +\int_{V_{ \pm}} d \vec{r}^{\prime} G^{ \pm}\left(\vec{r}, \vec{r}^{\prime}\right) \vec{\nabla} \delta \mu^{\prime} \cdot \frac{\vec{\nabla} \Gamma_{c}^{\prime}}{\Gamma_{c}^{\prime}}
\end{aligned}
$$

The last term in Eq. (C2) is $\mathcal{O}\left(\epsilon^{3}\right)$ and can be neglected. It is straightforward to repeat the calculations of Sec. III A for the velocity of the interface and for the chemical potential at the interface. All results remain the same except the diffusion constant $D_{c}$ has an obvious dependence on $\Gamma_{c}^{ \pm}$and

$$
\begin{aligned}
\mathcal{E}^{2}= & \Delta c \int_{0}^{\infty} \frac{d u}{\Gamma_{c}^{+}}\left[c_{0}^{\text {out }}(u)-c_{0}^{\text {in }}(u)\right]+\int_{-\infty}^{0} \frac{d u}{\Gamma_{c}^{-}}\left[c_{0}^{\text {in }}(u)\right. \\
& \left.-c_{0}^{\text {out }}(u)\right]^{2}+\int_{0}^{\infty} \frac{d u}{\Gamma_{c}^{+}}\left[c_{0}^{\text {in }}(u)-c_{0}^{\text {out }}(u)\right]^{2},
\end{aligned}
$$

and the interface position $u=0$ is determined by the solvability condition

$$
\int_{0}^{\infty} \frac{d u}{\Gamma_{c}^{+}}\left[c_{0}^{\text {out }}(u)-c_{0}^{\text {in }}(u)\right]=\int_{-\infty}^{0} \frac{d u}{\Gamma_{c}^{-}}\left[c_{0}^{\text {in }}(u)-c_{0}^{\text {out }}(u)\right] .
$$

[1] J. D. Gunton, M. San Miguel, and P. Sahni, in Phase Transitions and Critical Phenomena, edited by C. Domb and J. L. Lebowitz (Academic Press, London, 1983), Vol. 8, p. 267; A. J. Bray, Adv. Phys. 32, 357 (1994).

[2] J. S. Langer, Rev. Mod. Phys. 52, 1 (1980); E. Ben-Jacob, N. Goldenfeld, J. S. Langer, and G. Schön, Phys. Rev. A 29, 330 (1984); B. Caroli, C. Caroli, and B. Roulet, in Solids Far From Equilibrium, edited by G. Godrèche (Cambridge University Press, Cambridge, England, 1992); J. B. Collins and H. Le- vine, Phys. Rev. B 31, 6119 (1985).

[3] K. R. Elder, F. Drolet, J. M. Kosterlitz, and M. Grant, Phys. Rev. Lett. 72, 677 (1994); B. Grossmann, K. R. Elder, M. Grant, and J. M. Kosterlitz, ibid. 71, 3323 (1993); K. R. Elder, J. D. Gunton, and M. Grant, Phys. Rev. E 54, 6476 (1996); F. Drolet, K. R. Elder, Martin Grant, and J. M. Kosterlitz, ibid. 61, 6705 (2000).

[4] The wastefulness of simulating bulk regions can to a large extent be eliminated using adaptive grid methods, see, for ex- 
ample, N. Provatas, N. Goldenfeld, and J. Dantzig, Phys. Rev. Lett. 80, 3308 (1998).

[5] P. C. Hohenberg and B. I. Halperin, Rev. Mod. Phys. 49, 435 (1977).

[6] An English translation of van der Waals Ph.D. thesis is given by: J. S. Rowlinson, J. Stat. Phys. 20, 197 (1979).

[7] J. W. Cahn and J. E. Hilliard, J. Chem. Phys. 28, 258 (1958); 31, 688 (1959).

[8] S. M. Allen and J. W. Cahn, Acta Metall. 27, 1085 (1978).

[9] G. Caginalp and W. Xie, Phys. Rev. E 48, 1897 (1993).

[10] A. Karma and W-J. Rappel, Phys. Rev. E 53, 3017 (1996); 57, 4323 (1998).

[11] R. Almgren, SIAM (Soc. Ind. Appl. Math.) J. Appl. Math. 59, 2086 (1999).

[12] K. Kawasaki and T. Ohta, Prog. Theor. Phys. 67, 147 (1982); 68, 129 (1982); Physica A 118, 175 (1983); T. Ohta, D. Jasnow, and K. Kawasaki, Phys. Rev. Lett. 49, 1223 (1982); K. Kawasaki, M. C. Yalabik, and J. D. Gunton, Phys. Rev. A 17, 455 (1978); see also T. Rogers, Ph.D. thesis, University of Toronto, 1989.

[13] N. Goldenfeld, Lectures on Phase Transitions and the Renormalization Group (Addison-Wesley, Reading, MA, 1992).

[14] M. Plischke and B. Bergeersen, Equilibrium Statistical Physics (Prentice-Hall, Englewood Cliffs, NJ, 1989), Chap. 4.

[15] See, for example, L. Jörgenson, H. Guo, R. Harris, and M. Grant, Phys. Rev. E 48, 4592 (1993); L. Jörgenson, R. Harris, M. Grant, and H. Guo, ibid. 47, 1235 (1993); R. Harris, L. Jörgenson, and M. Grant, Phys. Rev. A 45, 1024 (1992), and references therein.

[16] I. M. Lifshitz, Zh. Éksp. Teor. Fiz. 42, 1354 (1962) [Sov. Phys. JETP 15, 939 (1962)].

[17] D. Turnbull, Trans. AIME 191, 661 (1952).

[18] R. Bausch, V. Dohm, H. K. Janssen, and R. K. P. Zia, Phys. Rev. Lett. 47, 1837 (1981); M. Grant and J. D. Gunton, Phys. Rev. B 28, 5496 (1983); T. Ohta, Ann. Phys. (NY) 158, 31 (1984).

[19] R. Pindak, C. Y. Young, R. B. Meyer, and N. A. Clark, Phys. Rev. Lett. 45, 1193 (1980).
[20] A. N. Kolmogorov, Bull. Acad. Sci. USSR, Mater. Ser. 1, 335 (1937); M. Avrami, J. Chem. Phys. 7, 1103 (1939); W. A. Johnson and A. Mehl, Trans. Am. Inst. Min., Metall. Pet. Eng. 135, 416 (1939).

[21] M. Kardar, G. Parisi, and Y. C. Zhang, Phys. Rev. Lett. 56, 889 (1986); see the derivation of the Kardar-Parisi-Zhang equation in B. Grossmann, H. Guo, and M. Grant, Phys. Rev. A 43, 1727 (1991).

[22] E. Ben-Jacob, N. Goldenfeld, B. G. Kotliar, and J. S. Langer, Phys. Rev. Lett. 53, 2110 (1984); see the review of Y. Pomeau and M. Ben Amar, in Solids Far From Equilibrium, edited by C. Godrèche (Cambridge University Press, Cambridge, England, 1991).

[23] R. Kobayashi, Physica D 63, 410 (1993).

[24] In two dimensions the tangential component of the time derivative can be written $v_{T} \partial / \partial s$, where $v_{T}$ accounts for flow of concentration (or $\psi$ ) along the interface. $v_{T}$ must be $\mathcal{O}(\epsilon)$ since it is zero when $\epsilon=0$. Furthermore, in the inner region $s$ is scaled with $\epsilon$ so $v_{T} \partial / \partial s \sim \mathcal{O}\left(\epsilon^{2}\right)$. When this term operates on $c$ or $\psi$ it becomes $\mathcal{O}\left(\epsilon^{3}\right)$, since both $c$ and $\psi$ are independent of $s$. The time derivative in the moving frame $\partial /\left.\partial t\right|_{(u, s)}$ is also at least $\mathcal{O}\left(\epsilon^{3}\right)$. This term accounts for the relaxation of fluctuations around the steady state profile not due to motion of the interface. For the concentration field fluctuations relax as $t$ $\sim(\text { length })^{2}$ which implies $\partial /\left.\partial t\right|_{(u, s)} \sim \mathcal{O}\left(\epsilon^{2}\right)$. As with the tangential component this term becomes $\mathcal{O}\left(\epsilon^{3}\right)$ when operating on $c$. For $\psi$, fluctuations relax exponentially fast and thus this term can be neglected.

[25] The last equality in Eqs. (49) and (50) was obtained using the relationships

$$
\frac{\partial}{\partial \bar{u}}\left(\frac{\partial f(c, \psi)}{\partial c}\right)=\frac{\partial^{2} f(c, \psi)}{\partial c^{2}} \frac{\partial c}{\partial \bar{u}}+\frac{\partial^{2} f(c, \psi)}{\partial c \partial \psi} \frac{\partial \psi}{\partial \bar{u}}=f^{(2,0)} \frac{\partial c}{\partial \bar{u}}+f^{(1,1)} \frac{\partial \psi}{\partial \bar{u}}
$$

and

$$
\frac{\partial}{\partial \bar{u}}\left(\frac{\partial f(c, \psi)}{\partial \psi}\right)=\frac{\partial^{2} f(c, \psi)}{\partial \psi^{2}} \frac{\partial \psi}{\partial \bar{u}}+\frac{\partial^{2} f(c, \psi)}{\partial c \partial \psi} \frac{\partial c}{\partial \bar{u}}=f^{(0,2)} \frac{\partial \psi}{\partial \bar{u}}+f^{(1,1)} \frac{\partial c}{\partial \bar{u}} .
$$

